\title{
THREE-DIMENSIONAL MHD SIMULATION OF THE CALTECH PLASMA JET EXPERIMENT: FIRST RESULTS
}

\author{
Xiang Zhai ${ }^{1}, \mathrm{Hui}_{\mathrm{LI}^{2}}{ }$, Paul M. Bellan ${ }^{1}$, and Shengtai $\mathrm{LI}^{3}$ \\ ${ }^{1}$ Applied Physics, California Institute of Technology, Pasadena, CA 91125, USA; xzhai@caltech.edu, pbellan@caltech.edu \\ 2 Theoretical Division, Los Alamos National Laboratory, Mail Stop B227, Los Alamos, NM 87545, USA; hli@ lanl.gov \\ ${ }^{3}$ Mathematical Modeling and Analysis, Los Alamos National Laboratory, Mail Stop B284, Los Alamos, NM 87545, USA; sli@lanl.gov \\ Received 2013 December 9; accepted 2014 June 22; published 2014 July 24
}

\begin{abstract}
Magnetic fields are believed to play an essential role in astrophysical jets with observations suggesting the presence of helical magnetic fields. Here, we present three-dimensional (3D) ideal MHD simulations of the Caltech plasma jet experiment using a magnetic tower scenario as the baseline model. Magnetic fields consist of an initially localized dipole-like poloidal component and a toroidal component that is continuously being injected into the domain. This flux injection mimics the poloidal currents driven by the anode-cathode voltage drop in the experiment. The injected toroidal field stretches the poloidal fields to large distances, while forming a collimated jet along with several other key features. Detailed comparisons between 3D MHD simulations and experimental measurements provide a comprehensive description of the interplay among magnetic force, pressure, and flow effects. In particular, we delineate both the jet structure and the transition process that converts the injected magnetic energy to other forms. With suitably chosen parameters that are derived from experiments, the jet in the simulation agrees quantitatively with the experimental jet in terms of magnetic/kinetic/inertial energy, total poloidal current, voltage, jet radius, and jet propagation velocity. Specifically, the jet velocity in the simulation is proportional to the poloidal current divided by the square root of the jet density, in agreement with both the experiment and analytical theory. This work provides a new and quantitative method for relating experiments, numerical simulations, and astrophysical observation, and demonstrates the possibility of using terrestrial laboratory experiments to study astrophysical jets.
\end{abstract}

Key words: galaxies: jets - ISM: jets and outflows - magnetohydrodynamics (MHD) - methods: numerical

Online-only material: color figures

\section{INTRODUCTION}

Magnetohydrodynamic (MHD) plasma jets exist in a wide variety of systems from terrestrial experiments to astrophysical objects, and have attracted substantial attention for decades. For example, energetic and usually relativistic jets are commonly observed originating from active galactic nuclei (AGNs), which are believed to be powered by supermassive black holes. AGN jets usually remain highly collimated for tens to hundreds of kiloparsecs from the host galaxy core (e.g., Ferrari 1998). It is generally accepted that AGN jets are powered by the central black hole accretion disk region. On a much smaller scale, stellar jets are believed to be an integral part of star formation with an active accretion disk surrounding a young star (e.g., Hartigan \& Hillenbrand 2009).

Despite our limited understanding of how the disks or central objects produce collimated jets, observational evidence has shown that magnetic fields are crucial in collimating and accelerating jets. Highly polarized synchrotron radiation is observed from both AGN jets and stellar jets, implying that jets have a strongly organized magnetic field. For example, the two lobes of T Tauri S, created by the interaction of a bipolar stellar jet with the remote interstellar medium (ISM), exhibit strong circularly polarized radio emission with opposite helicity (Ray et al. 1997). Large-scale magnetic fields from bipolar AGN jets also show transverse asymmetries (Clausen-Brown et al. 2011). These observations strongly suggest that a large-scale poloidal magnetic field, centered at the accretion disk or the central object, plays a crucial role in generating and propagating both AGN jets and stellar jets. A close look into the jet origin of M87 has found that the jet at 100 Schwarzschild radii is only weakly collimated (opening angle $\approx 60^{\circ}$ ), but becomes very collimated at larger distance (opening angle $<10^{\circ}$ ). This favors a magnetic collimation mechanism at $z>100$ Schwarzschild radii (Junor et al. 1999). The 3C31 jet and several other AGN jets exhibit a global kink-like $m=1$ instability or helical wiggles (Hardee 2008; Nakamura et al. 2007), implying the existence of a strong axial current along the jet, or, equivalently, a strong toroidal magnetic field around the jet. Here, we define the central axis along the jet as the $z$ axis of a cylindrical coordinate system. The $r$ and $z$ directions are called the "poloidal" direction and the azimuthal direction $\theta$ is called the "toroidal" direction. These facts suggest a $z$-pinch-type of collimation mechanism, in which the axial current $J_{z}$ and the associated azimuthal magnetic field $B_{\theta}$ generate a radial Lorentz force and squeeze the jet plasma against the pressure gradient at the central region of the jet.

The surprising similarities of astrophysical jets in morphology, kinetic behavior, and magnetic field configuration over vastly different scales have inspired many efforts to model these jets using ideal MHD theory. One important feature is that ideal MHD theory has no intrinsic scale. Therefore, an ideal MHD model is highly scalable and capable of describing a range of systems having many orders of magnitude difference in size. Ideal MHD theory assumes that the Lundquist number, a dimensionless measurement of plasma conductivity, to be infinite. This leads to the well-known "frozen-in" condition, wherein magnetic flux is frozen into the plasma and moves together with the plasma (Bellan 2006). Hence, the evolution of plasma material and magnetic field configuration is unified in ideal MHD. Blandford \& Payne (1982) developed a self-similar MHD model, in which a magnetocentrifugal mechanism accelerates plasma along poloidal field lines threading the accretion disk; the plasma is then collimated by a toroidal dominant magnetic field at larger distance. Lynden-Bell $(1996,2003)$ and Sherwin \& Lynden-Bell (2007) constructed an analytical magnetostatic MHD model where the upward flux of a dipole 
magnetic field is twisted relative to the downward flux. The height of the magnetically dominant cylindrical plasma grows in this configuration. The toroidal component of the twisted field is responsible for both collimation and propagation. The Lynden-Bell (1996, 2003) and Sherwin \& Lynden-Bell (2007) model and various following models (typically numerical simulations with topologically similar magnetic field configurations; e.g., Li et al. 2001, Lovelace et al. 2002, Li et al. 2006, Nakamura et al. 2008, and Xu et al. 2008), are called "magnetic tower" models. In these models, the large-scale magnetic fields are often assumed to possess "closed" field lines with both footprints residing in the disk. Because plasma at different radii on the accretion disk and in the corona have different angular velocity, the poloidal magnetic field lines threading the disk will become twisted up (Blandford \& Payne 1982; Lynden-Bell 1996, 2003; Sherwin \& Lynden-Bell 2007; Li et al. 2001; Lovelace et al. 2002), giving rise to the twist/helicity or the toroidal component of the magnetic fields in the jet. Faraday rotation measurements to 3C 273 show a helical magnetic field structure and an increasing pitch angle between toroidal and poloidal component along the jet (Zavala \& Taylor 2005). These results favor a magnetic structure suggested by magnetic tower models. Furthermore, it is (often implicitly) assumed that the mass loading onto these magnetic fields is small, so the communication by Alfvén waves is often fast compared to plasma flows.

These models have achieved various degrees of success and have improved understanding of astrophysical jets significantly. However, the limitations of astrophysical observation, e.g., mostly unresolved spatial features, passive observation, and impossibility of in-situ measurement, have imposed a natural limitation to these models. During the last decade, on the other hand, it has been realized that laboratory experiments can provide valuable insights for studying astrophysical jets. Laboratory experiments have the intrinsic value of elucidating key physical processes (especially those involving magnetic fields) in highly nonlinear systems. The relevance of laboratory experiments relies on the scalability of the MHD theory and the equivalence of differential rotation of the astrophysical accretion disk to voltage difference across the laboratory electrodes (at least in the magnetically dominated limit). The latter can be seen by considering Ohm's Law in ideal MHD theory, $\mathbf{E}+\mathbf{v} \times \mathbf{B}=0$; $\mathbf{E}$ is the electric field and $\mathbf{v}$ is the plasma velocity. The radial component of Ohm's Law is $E_{r}+v_{z} B_{\theta}-v_{\theta} B_{z}=0$. If we ignore the vertical motion $v_{z}$ of the accretion disk, it is seen that $E_{r}=v_{\theta} B_{z}$, i.e., an equivalent radial electric field is created by $\theta$ motion (rotation), and the spatial integration of this electric field gives the voltage difference at different radii. Such a voltage difference is relatively easy to create in lab experiments by applying a voltage across a coaxial electrode pair (See Section 3.3 for the discussion on the helicity). In addition, experimental jets are reproducible, parameterizable and in situ measurable. They automatically "calculate" the MHD equations and also "incorporate" non-ideal MHD plasma effects. Most importantly, the very fact that jets can be produced in the experiments strongly suggests there should be relatively simple unifying MHD concept characterizing AGN jets, stellar jets and experimental jets (Bellan et al. 2009).

The experiments carried out at Caltech and Imperial College have used pulse-power facilities to simulate "magnetic tower" astrophysical jets (e.g., Hsu \& Bellan 2002; Kumar \& Bellan 2009; Lebedev et al. 2005; Ciardi et al. 2007, 2009). The two experiments have topologically similar toroidal magnetic field configurations and plasma collimation mechanisms. However, in addition to the toroidal field, the Caltech plasma jets also have a poloidal magnetic field threading a co-planar coaxial plasma gun so the global field configuration is possibly more like a real astrophysical situation. Magnetically driven jets are produced by both groups, and the jets are collimated and accelerated in essentially the manner described by the magnetic tower models. Due to the lack of poloidal magnetic field, the plasma jets in the group at Imperial College undergo violent instability and break into episodic parts (magnetic bubbles). The Caltech jets remain very collimated and straight and undergo a kink instability when the jet length satisfies the classic Kruskal-Shafranov threshold (Hsu \& Bellan 2003, 2005). The Alfvénic and supersonic jets created by the Caltech group have relatively low thermal to magnetic pressure ratio $\beta \sim 0.1$ and large Lundquist number $S \sim 10-100$. Other features including flux rope merging, magnetic reconnection, Rayleigh-Taylor instability, and jetambient gas interaction are also produced (Hsu \& Bellan 2003, 2005; Yun \& Bellan 2010; Yun et al. 2007; Moser \& Bellan 2012a, 2012b). A detailed introduction to the Caltech jet experiments is given in Section 2.

Observation, analytical modeling, numerical simulation, and terrestrial experiments (laboratory astrophysics) are all crucial approaches for a better understanding of astrophysical jets. Compared to observation or analytical models, numerical simulation and terrestrial experiments share certain common features, such as the ability to deal with more complex structures and sophisticated behaviors, larger freedom in the parameter space compared to observation, and more resolution. However, cross-validation between numerical simulations and experiments has been very limited. Lab experiments can provide detailed validation for numerical models, while the numerical models can test the similarity between the terrestrial experimental jets and astrophysical jets.

We report here three-dimensional (3D) ideal MHD numerical studies that simulate the Caltech plasma jet experiment. The numerical model uses a modified version of a computational code (Li \& Li 2003) previously given by Li et al. (2006) for simulating AGN jets in the intra-cluster medium. Motivated by both observations and experiments, we adopt the approach that the jet has a global magnetic field structure and both poloidal and toroidal magnetic fields in the simulation are totally contained in a bounded volume. Following the approach in Li et al. (2006), the MHD equations are normalized to suit the experiment scale. An initial poloidal field configuration is chosen to simulate the experimental bias field configuration and the toroidal magnetic field injection takes a compact form to represent the electrodes. Detailed comparisons between simulation and experiment have been undertaken, addressing the collimation and acceleration mechanism, jet morphology, axial profiles of density and magnetic field, and the 3D magnetic field structure. The simulations have reproduced most salient features of the experimental jet quantitatively, with discrepancies generally less than a factor of three for key quantities. The conversion of magnetic to kinetic energy from jet base to jet head is examined in the simulation and compared to the experiment. As a result, a Bernoulli-like equation, stating that the sum of kinetic and toroidal magnetic field energy is constant along the axial extent of the jet, is validated by analytical modeling, laboratory experiment and the numerical simulation.

The paper is organized as follows: In Section 2, we introduce the Caltech plasma jet experiment and demonstrate that the global behavior of the experimental jets can be described by ideal MHD theory. In Section 3, we describe the approach and 


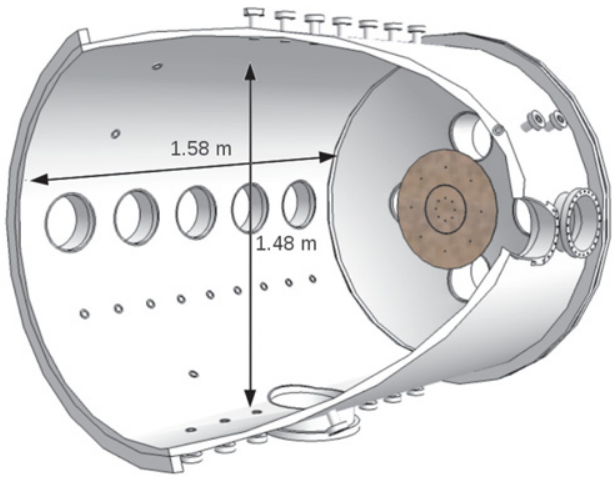

$\mathrm{H}$ jet shot\#11497
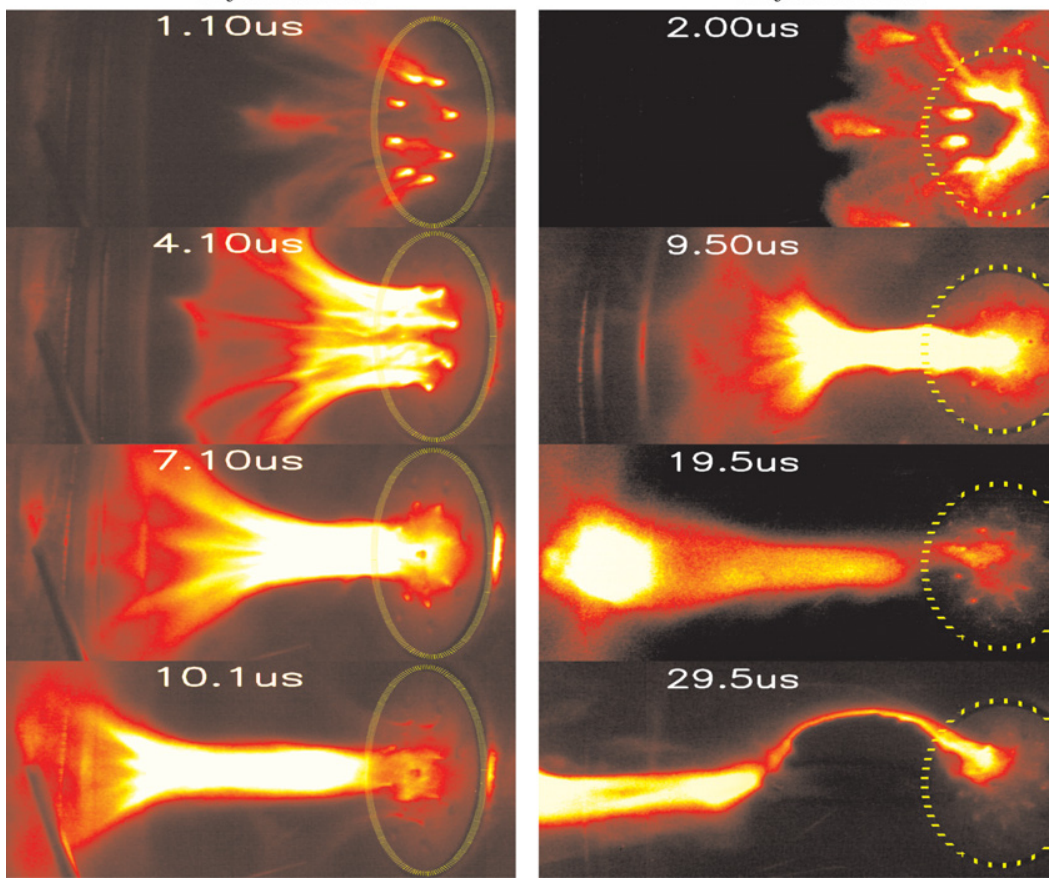

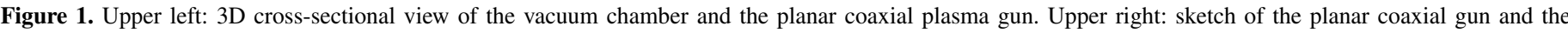

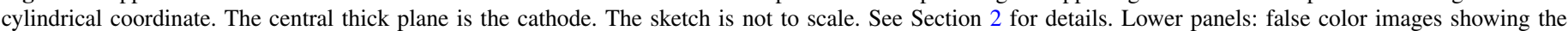

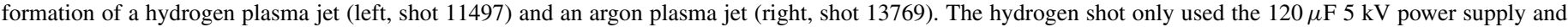

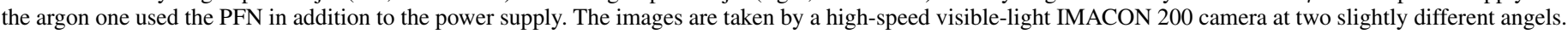
The dotted circles at the right of each frame represent the $10 \mathrm{~cm}$ radius central cathode.

(A color version of this figure is available in the online journal.)

configuration of our simulation, and show that the compact toroidal magnetic field injection method used in the simulation is equivalent to the energy and helicity injection through the electrodes used in the experiment. In Section 4.1, we present the simulation results of a typical run, and compare these results with experimental measurements. In Section 4.2, we perform multiple simulations with different toroidal injection rates and examine the jet velocity dependence on poloidal current. These results together with experimental measurements confirm the MHD Bernoulli equation and the magnetic to kinetic energy conversion in the MHD-driven plasma jet. Section 5 discusses the sensitivity of the simulation results to initial and injection conditions. Conclusions and discussions are given in Section 6.

\section{CALTECH PLASMA JET EXPERIMENT}

The Caltech experimental plasma jet is generated using a planar magnetized coaxial plasma gun mounted at one end of a $1.48 \mathrm{~m}$ diameter, $1.58 \mathrm{~m}$ long cylindrical vacuum chamber (sketch in Figure 1). The vacuum pressure is $\sim 10^{-7}$ torr, corresponding to a background particle density of $3 \times 10^{15} \mathrm{~m}^{-3}$. The plasma gun has a $19.1 \mathrm{~cm}$ diameter disk-shaped cathode and a co-planar annulus-shaped anode with inner diameter $d=20.3 \mathrm{~cm}$ and outer diameter $D=51 \mathrm{~cm}$. The electrode plane is defined as $z=0$ and the central axis is the $z$ axis. At time $t=-10 \mathrm{~ms}$, a circular solenoid coil behind the cathode electrode generates a dipole-like poloidal background magnetic field for $\sim 20 \mathrm{~ms}$, referred to as the bias field. The total poloidal field flux is about $1.5 \mathrm{mWb}$. At $t=-1$ to $-5 \mathrm{~ms}$, neutral gas is puffed into the vacuum chamber through eight evenly spaced holes at $r=5 \mathrm{~cm}$ on the cathode and eight holes at $r=18 \mathrm{~cm}$ on the anode at the same azimuthal angles. At $t=0$, a $120 \mu \mathrm{F} 5 \mathrm{kV}$ high-voltage capacitor is switched across the electrodes. This breaks down the neutral gas into eight arched plasma loops spanning from the anode to the cathode following the bias poloidal field lines. At $0.6 \mu$ s after breakdown, a $4 \mathrm{kV}$ pulse forming network (PFN) supplies additional energy to the plasma and maintains a total poloidal current at 60-80 kA for $\approx 40 \mu$ s. A typical current and voltage measurement is given in Figure 3. 
Diagnostic instrumentation includes a high-speed visiblelight IMACON 200 camera, a 12-channel spectroscopic system (Yun \& Bellan 2010), a He-Ne interferometer perpendicular to the jet (Kumar \& Bellan 2006), a 20 channel 3D magnetic field probe array (MPA) along the $r$ direction with adjustable $z$ and $\sim 1 \mu$ s response time (Romero-Talamás et al. 2004), another similar MPA along the $z$ axis, a fast ion gauge, a Rogowski coil and a Tektronix high-voltage probe.

Fast ion gauge measurements show that the neutral particle number density immediately before the plasma breakdown is $10^{19}-10^{20} \mathrm{~m}^{-3}$ (Moser 2012; Moser \& Bellan 2012b). When the eight arched plasma loops are initially formed, the poloidal current and poloidal magnetic field in the loops are parallel to each other. However, the plasma is not a force-free system because of the toroidal magnetic field associated with the poloidal current. The inner segments of the eight arched loops, carrying parallel current from the anode to the cathode, mutually attract each other by the Lorentz force and merge into a single collimated plasma tube along the $z$ axis. A 10-fold density amplification in the jet due to collimation is observed by Stark broadening and interferometer measurements; these show the typical density of the collimated jet is $10^{22}-10^{23} \mathrm{~m}^{-3}$ (Yun et al. 2007; Kumar \& Bellan 2009; Yun \& Bellan 2010). The poloidal magnetic field strength in the plasma is also amplified from $<0.05 \mathrm{~T}$ to $\sim 0.2 \mathrm{~T}$, indicating that the field is frozen into the plasma and is collimated together with the plasma. This amplification of the magnetic field strength has also been observed spectroscopically (Shikama \& Bellan 2013). The thermal pressure and axial magnetic field pressure $B_{z}^{2} /\left(2 \mu_{0}\right)$ increase until they balance the radial Lorentz force and lead to a nearly constant jet radius of $2-5 \mathrm{~cm}$ (Figure 1) and a toroidal magnetic field $B_{\theta} \sim 0.1-0.5 \mathrm{~T}$ (see experimental measurements in Figure 10). This radial equilibrium is gradually established from small to large $z$, resulting in an MHD pumping mechanism that accelerates the plasma toward the $+z$ direction to form a jet. The typical jet velocity is $10-20 \mathrm{~km} \mathrm{~s}^{-1}$ for argon, $30-40 \mathrm{~km} \mathrm{~s}^{-1}$ for nitrogen and $\sim 50 \mathrm{~km} \mathrm{~s}^{-1}$ for hydrogen (Kumar \& Bellan 2009). The plasma jet, as a one-end-free current-conducting plasma tube, undergoes a kink instability when its length grows long enough to satisfy the classical Kruskal-Shafranov kink threshold (Hsu \& Bellan 2003, 2005). When the kink grows exponentially fast and accelerates the plasma laterally away from the central axis, an effective gravitational force is experienced by the accelerating plasma jet. At the inner boundary of the kinked jet, where this effective gravity points from the displaced jet (dense plasma) to the $z$ axis (zero-density vacuum), a Rayleigh-Taylor instability occurs (Moser \& Bellan 2012a). The Rayleigh-Taylor instability eventually leads to a fast magnetic reconnection and destroys the jet structure. The jet lifetime is $\sim 10 \mu$ s for hydrogen, 20-30 $\mu$ s for nitrogen and 30-40 $\mu$ s for argon. Because heating is not important during this short, transient lifetime, the plasma remains at a relatively low temperature $T_{e} \sim 2 \mathrm{eV}$ inferred from spectroscopic measurements (Yun \& Bellan 2010). Under typical experiment plasma conditions, the temperature relaxation time between electrons and ions is about $100 \mathrm{~ns}$, less than $1 \%$ of the jet lifetime. Therefore, $T_{i} \approx T_{e} \sim 2 \mathrm{eV}$. At this temperature, the plasma is essentially $100 \%$ singly ionized according to the Saha-Boltzmann theory, which is also confirmed by spectroscopic measurements (e.g., Yun \& Bellan 2010; Hsu $\&$ Bellan 2003). Figure 1 shows how the plasma is initially generated as eight arched loops, which then merge into one collimated jet. The jet then undergoes a kink instability when its length exceeds $\sim 30-40 \mathrm{~cm}$. For the current experiment config- uration, the radius-length ratio of the jet in the final stage is about $1: 10$.

For a typical experimental plasma with $n_{e}=10^{22} \mathrm{~m}^{-3}$, $T_{e}=T_{i}=2 \mathrm{eV}, B=0.2 \mathrm{~T}$ and ion mass $\mu \equiv m_{i} / m_{H}$, the Debye length $\lambda_{D} \approx 10^{-7} \mathrm{~m}$, the ion gyroradius $r_{i} \approx 0.7 \sqrt{\mu} \mathrm{mm}$ and the ion skin depth $d_{i} \approx 2 \sqrt{\mu}$ mm are all significantly smaller than the length/radius of the experimental jet. The typical thermal to magnetic energy density ratio is $\beta \approx 0.1-1$, showing that the magnetic field is essential to the jet dynamics. Despite its relatively low temperature, the plasma has sufficiently high conductivity so that the Lundquist and magnetic Reynolds numbers $S \sim R_{m} \gtrsim 10^{2} \times(L / 0.3 \mathrm{~m}) / \sqrt{\mu}$ are both much greater than one with $L \sim 0.3 \mathrm{~m}$, where $L$ is the length scale of phenomena of interest. Therefore, ideal MHD theory can describe jet global dynamics, such as collimation, acceleration, and kinking (Hsu \& Bellan 2003, 2005; Yun et al. 2007; Yun \& Bellan 2010; Kumar \& Bellan 2009; Kumar 2009), and magnetic field diffusion is negligible during the jet dynamics. The kinked jet image in Figure 1 shows that the magnetic field is frozen into the plasma, consistent with ideal MHD theory. Hence, the collimation of the bright plasma shown in Figure 1 also demonstrates the collimation of the magnetic field. The arched loops merging and the secondary Rayleigh-Taylor instability, on the other hand, involve ion skin depth length scale phenomena, that are smaller than can be described by MHD theory (Moser \& Bellan 2012a).

\section{NUMERICAL MHD SIMULATIONS}

Discussion in this paper is restricted to the global axisymmetric behaviors of the jet, such as collimation and acceleration. Non-axisymmetric instabilities will be discussed in future publications. In this section, we prescribe appropriate initial and boundary conditions used to solve the ideal MHD equations numerically for the Caltech plasma jet experiment.

\subsection{Normalization and Equations}

Number density, length, and velocity are scaled to nominal reference values. In particular, density is normalized to $n_{0}=$ $10^{19} \mathrm{~m}^{-3}$, lengths are normalized to $R_{0}=0.18 \mathrm{~m}$ (radial position of the outer gas feeding holes of the plasma gun in the experiment), and velocities are normalized to the ion sound speed $C_{s 0}=\sqrt{2 k T / m_{i}}=1.96 \times 10^{4} \sqrt{m_{H} / m_{i}} \mathrm{~m} \mathrm{~s}^{-1}$ (with temperature $2 \mathrm{eV}$ ). All other quantities are normalized to reference values derived from these three nominal values and ion mass $m_{i}$. Table 1 lists the derivation and the normalization values adopted in the experimental hydrogen/argon jet simulation and the AGN jet simulation by Li et al. (2006). SI units are used for the lab experiment while cgs units are used for the AGN jet in order to facilitate comparison to respective experimental and astrophysical literature.

The dimensionless ideal MHD equations, normalized to the quantities given in Table 1, are

$$
\begin{gathered}
\frac{\partial \rho}{\partial t}+\nabla \cdot(\rho \mathbf{v})=0 \\
\frac{\partial(\rho \mathbf{v})}{\partial t}+\nabla \cdot\left(\rho \mathbf{v v}+P_{g} \overleftrightarrow{\mathbf{I}}+P_{B} \overleftrightarrow{\mathbf{I}}-\mathbf{B B}\right)=0 \\
\frac{\partial e}{\partial t}+\nabla \cdot\left[\left(e+P_{g}+P_{B}\right) \mathbf{v}-\mathbf{B}(\mathbf{v} \cdot \mathbf{B})\right]=\dot{e}_{i n j} \\
\frac{\partial \mathbf{B}}{\partial t}-\nabla \times(\mathbf{v} \times \mathbf{B})=\dot{\mathbf{B}}_{\mathrm{inj}}
\end{gathered}
$$


Table 1

Normalization Units for the Experimental H/Ar Jet Simulation and the AGN Jet Simulation

\begin{tabular}{|c|c|c|c|c|}
\hline Quantity Unit & Quantity Symbols & $\mathrm{H}(\mu=1)$ & $\operatorname{Ar}(\mu=40)$ & AGN Jet $(\mu=1)$ \\
\hline Length & $R_{0}$ & $0.18 \mathrm{~m}$ & $0.18 \mathrm{~m}$ & $15 \mathrm{kpc}$ \\
\hline Number density & $n_{0}$ & $10^{19} \mathrm{~m}^{-3}$ & $10^{19} \mathrm{~m}^{-3}$ & $3 \times 10^{-3} \mathrm{~cm}^{-3}$ \\
\hline Speed & $C_{s 0}$ & $1.96 \times 10^{4} \mathrm{~m} \mathrm{~s}^{-1}$ & $3.1 \times 10^{3} \mathrm{~m} \mathrm{~s}^{-1}$ & $1.16 \times 10^{8} \mathrm{~cm} \mathrm{~s}^{-1}$ \\
\hline Ion weight & $\mu=m_{i} / m_{H}$ & 1 & 40 & 1 \\
\hline Time & $t_{0}=R_{0} / C_{s 0}$ & $9.2 \mu \mathrm{s}$ & $58.2 \mu \mathrm{s}$ & $1.3 \times 10^{7} \mathrm{yr}$ \\
\hline Mass density & $\rho_{0}=n_{0} m_{i} / 2$ & $8.4 \times 10^{-9} \mathrm{~kg} \mathrm{~m}^{-3}$ & $3.3 \times 10^{-7} \mathrm{~kg} \mathrm{~m}^{-3}$ & $2.5 \times 10^{-30} \mathrm{~g} \mathrm{~cm}^{-3}$ \\
\hline Pressure & $p_{0}=\rho_{0} C_{s 0}^{2}$ & $3.2 \mathrm{pa}$ & $3.2 \mathrm{pa}$ & $3.4 \times 10^{-11} \mathrm{erg} \mathrm{cm}^{-3}$ \\
\hline Temperature & $k_{B} T=m_{i} C_{s 0}^{2} / 2$ & $2 \mathrm{eV}$ & $2 \mathrm{eV}$ & $7 \mathrm{keV}$ \\
\hline Energy & $E_{0}=p_{0} R_{0}^{3}$ & $0.0187 \mathrm{~J}$ & $0.0187 \mathrm{~J}$ & $3.4 \times 10^{57} \mathrm{erg}$ \\
\hline Power & $P_{0}=E_{0} / t_{0}$ & $2.0 \times 10^{3}$ Watt & 321 Watt & $2.6 \times 10^{50} \mathrm{erg} \mathrm{yr}^{-1}$ \\
\hline Magnetic field & $B_{0}=\sqrt{\mu_{0} p_{0}}$ & $0.002 \mathrm{~T}$ & $0.002 \mathrm{~T}$ & $2 \times 10^{-5}$ Gauss \\
\hline Magnetic flux & $\Psi_{0}=B_{0} R_{0}^{2}$ & $0.0648 \mathrm{mWB}$ & $0.0648 \mathrm{mWB}$ & $4.4 \times 10^{40} \mathrm{G} \mathrm{cm}^{2}$ \\
\hline Current density & $J_{0}=B_{0} /\left(\mu_{0} R_{0}\right)$ & $8.871 \times 10^{3} \mathrm{~A} \mathrm{~m}^{-2}$ & $8.871 \times 10^{3} \mathrm{~A} \mathrm{~m}^{-2}$ & $3.5 \times 10^{-28} \mathrm{~A} \mathrm{~cm}^{-2}$ \\
\hline Current & $I_{0}=J_{0} R_{0}^{2}$ & $2.874 \times 10^{2} \mathrm{~A}$ & $2.874 \times 10^{2} \mathrm{~A}$ & $7.6 \times 10^{17} \mathrm{~A}$ \\
\hline Voltage & $V_{0}=P_{0} / I_{0}$ & $7.07 \mathrm{~V}$ & $1.118 \mathrm{~V}$ & $1.1 \times 10^{18} \mathrm{~V}$ \\
\hline
\end{tabular}

where all the dimensionless variables have their conventional meaning. The momentum equation and the energy equation have been written in the form of conservation laws. We assume the same ion/electron temperature $T=T_{i}=T_{e}$. The particle number density $n=2 n_{e}=2 n_{i}$ is used assuming singly ionized plasma. The ionization status is assumed to be time-independent. The equation of state for an ideal gas with adiabatic index $\gamma=5 / 3$ is used. The gas pressure $P_{g}=n_{i} k_{B} T_{i}+n_{e} k_{B} T_{e}=$ $n k_{B} T$ is then related to the thermal energy density by $e_{\text {thermal }}=$ $P_{g} /(\gamma-1)$. The magnetic pressure $P_{B}$, or magnetic energy density $e_{B}$, is $P_{B}=e_{B}=B^{2} /\left(2 \mu_{0}\right)$ and the total energy density is $e \equiv \rho v^{2} / 2+P_{g} /(\gamma-1)+P_{B}$.

An injection term $\dot{\mathbf{B}}_{\text {inj }}$ is added to the induction equation. The associated dimensionless energy density injection is

$$
\dot{e}_{\text {inj }}=\dot{\mathbf{B}}_{\text {inj }} \cdot \mathbf{B},
$$

where $\mathbf{B}$ is the magnetic field.

Simulations are performed in a 3D Cartesian coordinate system $\{x, y, z\}$ using the 3D MHD code as part of the Los Alamos COMPutational Astrophysics Simulation Suite (LACOMPASS; Li \& Li 2003). This package was previously used for simulating AGN jets (e.g., Li et al. 2006). The solving domain is a cube $\left[-4 R_{0}, 4 R_{0}\right]^{3}=[-0.72 \mathrm{~m}, 0.72 \mathrm{~m}]^{3}$, similar to the vacuum chamber size in the experiment. Each Cartesian axis is discretized into 800 uniformly spaced grids, giving a total of $5.12 \times 10^{8}$ grid points. The spatial resolution $\Delta x=$ $8 R_{0} / 800=1.8 \mathrm{~mm}$ in the simulation is significantly greater than the Debye length, and is similar to the ion gyroradius and the ion skin depth of the plasma jet in the experiment. A typical run takes 5 to $24 \mathrm{hr}$ on the Los Alamos National Lab Turquoise Network using 512 processors.

In contrast to the experiment where the jet exists only for positive $z$, the simulation has a mirrored plasma jet in the negative $z$ direction so as to have a bipolar system centered at $z=0$ plane. The solving domain contains plasma only and has no plasma-electrode interaction region. Non-reflecting outflow boundary conditions are imposed at the boundaries (large $x, y$ or $z$ ). The MHD equations are solved in Cartesian coordinates so that no computational singularity exists at the origin.

\subsection{Initial Condition}

\subsubsection{Initial Global Poloidal Magnetic Field}

It is generally believed that the poloidal and toroidal magnetic components evolve together under the dynamo processes in the accretion disk and surrounding corona. However, when the poloidal component varies slower than the toroidal component, it is possible to treat the two components separately. In LyndenBell (1996, 2003), a poloidal field is assumed to be pre-existing, and the toroidal field is generated by twisting the upward flux relative to downward flux. During this process, the poloidal flux remains constant while toroidal field is enhanced with the increase of number of turns (helicity). These processes are realized equivalently in the lab experiment, where an initial dipole poloidal field is first generated by an external coil, and then helicity is increased by injecting poloidal current. In the simulation, an initial dipole poloidal magnetic field is similarly imposed, given by

$$
\Psi_{\mathrm{pol}}(r, z) \equiv 2 \pi \alpha_{p} \frac{r^{2}}{\left(l^{2}+a_{0}^{2}\right)^{3 / 2}} e^{-l^{2}}
$$

where $a_{0} \equiv 0.623 R_{0}=11.2 \mathrm{~cm}\left(R_{0}=0.18 \mathrm{~m}\right.$, see Table 1$)$ and $l \equiv \sqrt{r^{2}+z^{2}}$ is the distance from the origin. This configuration is topologically similar to the initial poloidal flux $\Psi_{\text {pol }}=r^{2} e^{-l^{2}}$ adopted by Li et al. (2006). By default, simulation equations/variables will be written in a dimensionless form with reference units given in Table 1. For example, Equation (3) is the dimensionless version of $\Psi_{\text {pol }}(r, z)=$ $2 \pi \alpha_{p} B_{0} R_{0}^{2}\left(r / R_{0}\right)^{2} /\left[\left(l / R_{0}\right)^{2}+\left(a_{0} / R_{0}\right)^{2}\right]^{3 / 2} e^{-l^{2} / R_{0}^{2}}$, where $B_{0}$ and $R_{0}$ are given in Table 1 . Compared to the ideal infinitesimal magnetic dipole flux $\Psi \propto r^{2} / l^{3}, \Psi_{\text {pol }}$ contains a constant factor $a_{0}$ to make the dipole source finite; it also has an exponential decay at large distance so that the initial field vanishes at the solving domain boundaries. At small $r$ and $z, \Psi_{\text {pol }} \propto r^{2}$ hence $B_{z}$ is nearly constant. $a_{0}$ is selected so that $\Psi_{\mathrm{pol}}\left(r=r_{1}, z=0\right)=\Psi_{\mathrm{pol}}\left(r=r_{2}, z=0\right)$, where $r_{1}=0.278 \Rightarrow 5 \mathrm{~cm}$ and $r_{2}=1 \Rightarrow 18 \mathrm{~cm}$ corresponding to the radii of the inner and outer gas lines in the experiment. The dimensionless parameter $\alpha_{p}$ quantifies the strength of the flux. 
The vector potential can be selected to be $\mathbf{A}=\left(\Psi_{\mathrm{pol}} /(2 \pi r)\right) \hat{\theta}$. The initial poloidal field is

$$
\begin{gathered}
\mathbf{B}_{\mathrm{pol}}=\nabla \times \mathbf{A}=\frac{1}{2 \pi} \nabla \Psi_{\mathrm{pol}} \times \nabla \theta \\
\Rightarrow\left\{\begin{array}{l}
B_{r}=\frac{\alpha_{p} z r e^{-l^{2}}}{\left(l^{2}+a_{0}^{2}\right)^{5 / 2}}\left(3+2 a_{0}^{2}+2 l^{2}\right) \\
B_{z}=\frac{\alpha_{p} e^{-l^{2}}}{\left(l^{2}+a_{0}^{2}\right)^{5 / 2}}\left[2\left(1-r^{2}\right)\left(l^{2}+a_{0}^{2}\right)-3 r^{2}\right],
\end{array}\right.
\end{gathered}
$$

where $\hat{\theta}$ is the azimuthal unit vector and $\nabla \theta=\hat{\theta} / r$. The total poloidal flux is

$$
\Psi_{0, p o l} \equiv \Psi_{\mathrm{pol}}\left(r_{o}, 0\right)=2.448 \alpha_{p} \Rightarrow 0.1586 \alpha_{p} \mathrm{mWb},
$$

where $r_{o}=0.5667 \Rightarrow 10.20 \mathrm{~cm}$ is the position of the null of the initial poloidal field, i.e., $B_{z}\left(r_{o}, 0\right)=0$. The first frame of Figure 4 shows the flux contours of the initial poloidal field in the $r z$ plane.

The toroidal current associated with the poloidal field is

$$
J_{\theta}=\partial_{z} B_{r}-\partial_{r} B_{z}=-\alpha_{p} \frac{r e^{-l^{2}}}{\left(l^{2}+a_{0}^{2}\right)^{7 / 2}} \cdot g(l),
$$

where

$$
g(l)=4 l^{6}+\left(8 a_{0}^{2}+2\right) l^{4}+4 a_{0}^{2}\left(a_{0}^{2}-2\right) l^{2}-5 a_{0}^{2}\left(a_{0}^{2}+3\right) .
$$

Simple calculation shows that $l_{0}=0.9993 \approx 1$ is the only zero point of $g(l)$ and $g(l)<0$ for $0 \leqslant l<l_{0}$ and $g(l)>0$ for $l>l_{0}$.

\subsubsection{Initial Mass Distribution}

In the experiment, plasma is initially created following the path of initial poloidal field lines (see Figure $1 \mathrm{H}$ jet at $1.1 \mu \mathrm{s}$ and $\mathrm{Ar}$ jet at $2.0 \mu \mathrm{s}$ ), i.e., the plasma is distributed around the $\Psi_{\mathrm{pol}}(r, z)=\Psi_{0}$ surface. Here, $\Psi_{\mathrm{pol}}(r, z)$ is the initial poloidal flux function (Equation (3)) and $\Psi_{0} \equiv \Psi_{\mathrm{pol}}\left(r_{1}, 0\right)=\Psi_{\mathrm{pol}}\left(r_{2}, 0\right)$ is the flux contour connecting the inner $\left(r_{1}=5 \mathrm{~cm}\right)$ and outer $\left(r_{2}=18 \mathrm{~cm}\right)$ gas feeding holes. A possible choice for the initial mass distribution function in the simulation is $n_{\text {init }} \sim \exp \left[-\delta\left(\Psi_{\text {pol }}(r, z)-\Psi_{0}\right)^{2}\right]$.

Note that this initial distribution has low plasma density on the axis. In the experiment, fast magnetic reconnection occurs as the eight arched loops merge into one. This allows the plasma and magnetic field to fill in the central region. The ideal MHD simulation, however, lacks the capability to simulate the fast magnetic reconnection, and hence cannot accurately describe the merging process. As a compromise, we start the simulation immediately after the merging process but before the collimation and propagation processes. We therefore choose a simple form topologically similar to the contour $\Psi_{\mathrm{pol}}(r, z)=\Psi_{0}$ but without the central hollow region, namely

$$
n_{\text {init }}(r, z)=1+n_{\text {init }, 0} \cdot e^{-l^{2}} \cdot e^{-\delta\left[(r-1 / 2)^{2}+z^{2}-1 / 4\right]^{2}} .
$$

The first term 1 corresponds to a background particle number density $10^{19} \mathrm{~m}^{-3}$. This is $\sim 10^{3}$ times more dense than the background in the experiment, but still $\sim 10^{-3}$ less dense than the plasma jet. $n_{\text {init, } 0}$ is the assumed initial plasma number density. The $e^{-\delta\left[(r-1 / 2)^{2}+z^{2}-1 / 4\right]^{2}}$ term states that the plasma is initially distributed over a torus surface $(r-1 / 2)^{2}+z^{2}=1 / 4$ connecting $r=0$ and $r=1=18 \mathrm{~cm}$ at mid plane. The torus surface is roughly parallel to the initial poloidal flux surface $\Psi_{\mathrm{pol}}(r, z)=\Psi_{0}$, but without the central hole. The $e^{-l^{2}}$ term assures that the initial plasma is localized around the origin. Using this distribution, the central region $r \simeq 0$ in the simulation is initially filled with dense plasma.

\subsection{Helicity and Energy Injection}

\subsubsection{Compact Injection Near the $z=0$ Plane}

Toroidal magnetic flux is continuously injected into the simulation system, in order to replicate the energy and magnetic injection through the electrodes in the experiment. The helicity conservation equation in an ideal MHD plasma with volume $\mathcal{V}$ is

$$
\frac{d K_{\mathrm{rel}}}{d t}=-\int_{\partial \mathcal{V}}(2 V \mathbf{B}) \cdot d \mathbf{S}=2 \Psi_{\mathrm{pol}} \cdot \frac{\partial(I L)}{\partial t},
$$

where $K_{\text {rel }}$ is the relative magnetic helicity, $\partial \mathcal{V}$ is the boundary of the volume and the area $d \mathbf{S}$ is normal to the boundary, $V$ is the electrode voltage, $I$ is the total current through the plasma, and $L$ is the plasma self-inductance across the electrodes (Finn \& Antonsen 1985; Berger 1999; Bellan 2000; Kumar 2009). The electrode surface in the experiment is the effective $\partial \mathcal{V}$. When a poloidal magnetic field is present, Equation (10) states that magnetic helicity injection can be realized either by maintaining a non-zero voltage across the electrodes, or by increasing the poloidal current/toroidal field in the plasma. In the experiment, these two methods are essentially equivalent. Meanwhile, magnetic energy is also injected into the plasma by $\dot{E}=P=I V=I d \Psi_{\text {tor }} / d t$, where $\Psi_{\text {tor }}$ is the toroidal magnetic flux. Since neither electric field nor potential is explicitly used in the simulation, we choose the second method to inject helicity. Thus we inject toroidal magnetic field into the system to increase the poloidal current and the magnetic helicity. The toroidal field injection term in Equation (1d) is defined as

$$
\dot{\mathbf{B}}_{\text {inj }} \equiv \gamma_{b}(t) \mathbf{B}_{\text {tor }},
$$

where $\gamma_{b}(t)$ is the injection rate and

$$
\mathbf{B}_{\mathrm{tor}}=\frac{f\left(\Psi_{\mathrm{pol}}\right)}{2 \pi r} e^{-A z^{2}} \hat{\theta}=\frac{1}{2 \pi} f\left(\Psi_{\mathrm{pol}}\right) e^{-A z^{2}} \nabla \theta
$$

is a pure toroidal field. The localization factor $A$ is a large positive number so that toroidal field injection is localized near the $z=0$ plane. $f\left(\Psi_{\mathrm{pol}}\right)$ is an analytical function of $\Psi_{\mathrm{pol}}$ and following the magnetic tower model used in Li et al. (2006), we choose $f\left(\Psi_{\mathrm{pol}}\right)=\alpha_{t} \Psi_{\mathrm{pol}}$ so that

$$
\begin{aligned}
\mathbf{B}_{\mathrm{tor}} & =\alpha_{t} \alpha_{p} \frac{r}{\left(l^{2}+a_{0}^{2}\right)^{3 / 2}} e^{-l^{2}} e^{-A z^{2}} \\
& =\alpha_{t} \alpha_{p} \frac{r}{\left(r^{2}+z^{2}+a_{0}^{2}\right)^{3 / 2}} e^{-r^{2}-(A+1) z^{2}} .
\end{aligned}
$$

The poloidal current associated with this toroidal field is

$$
\begin{aligned}
\mathbf{J}_{\mathrm{pol}} & =\nabla \times \mathbf{B}_{\mathrm{tor}}=\frac{1}{2 \pi} \nabla\left(\alpha_{t} \Psi_{\mathrm{pol}} e^{-A z^{2}}\right) \times \nabla \theta \\
& =\alpha_{t} e^{-A z^{2}} \mathbf{B}_{\mathrm{pol}}+\frac{\alpha_{t} \Psi_{\mathrm{pol}}}{\pi r} A z e^{-A z^{2}} \hat{r}
\end{aligned}
$$

where $\nabla \times \nabla \theta=0$ and Equation (4) are used. 
At $z=0, B_{\text {tor }}=\alpha_{t} \Psi_{\text {pol }} /(2 \pi r)$. Therefore, the net poloidal current within radius $r$ is $2 \pi r B_{\text {tor }}=\alpha_{t} \Psi_{\text {pol }}$. Using Equation (6), the total positive poloidal current associated with $B_{\text {tor }}$ is

$$
I_{\mathrm{pol}}=2.488 \alpha_{t} \alpha_{p} \Rightarrow 0.704 \alpha_{t} \alpha_{p} \mathrm{kA}
$$

The localization factor $A$ has no impact on the total poloidal current.

It is important to point out that the field injection term in the induction Equation (1d) is a compromise used to avoid having a plasma-electrode interaction boundary condition. Theoretically, Equation (1d) is not physically correct because of the injection term. However, because the localization factor $A$ is a large positive number, the magnetic energy of $\mathbf{B}_{\text {tor }}$ decreases rapidly with $z$. Therefore, the "unphysical" region is very localized to the vicinity of the $z=0$ plane. In particular, using $A=9$, the total toroidal magnetic energy at the $z=0.307 \Rightarrow 5.5 \mathrm{~cm}$ plane is only $10 \%$ of the total planar magnetic energy at the $z=0$ plane. The toroidal magnetic flux within $|z|<0.307$ contributes $87 \%$ of the total toroidal flux, although the volume is only $7.7 \%$ of the total simulation domain. We define $z_{\text {foot }} \equiv 0.307$, so the region where $|z|<z_{\text {foot }}$ is the "engine region" where most of the energy injection is enclosed, and the region outside the engine region $\left(|z|>z_{\text {foot }}\right)$ is the "jet region" where unphysical toroidal field injection does not occur. In the engine region, the toroidal magnetic field is directly added to the existing configuration by the modified induction equation (Equation (1d)). The injection also adds magnetic helicity, poloidal current and magnetic energy. In the jet region, on the other hand, this direct injection is negligible so the ideal MHD laws hold almost perfectly. The helicity, current, and energy enter the jet region with the plasma flow.

In the simulations presented here, we use $A=9$. Although the choice of $A$ is somewhat arbitrary, in general, $A$ needs to be sufficient large to localize the engine region to the vicinity of the $z=0$ plane. This compact engine region serves as an effective plasma-electrode interface, and leaves most of the simulation domain described by the correct induction equations (i.e., no artificial injection). If a small $A$ were used, injection would occur globally. There would then be a large amount of energy directly added to remote regions with low density plasma. A magnetized shock would then arise and dissipate injected energy. Using a large $A$ guarantees that the magnetic field is mostly frozen into the dense plasma. However, $A$ should be not too large in our simulation, since otherwise numerical instability and error would occur because of excessive gradients.

The process of helicity/energy injection in the simulation is not exactly the same as in the experiments or the astrophysical case. In the experiment, a non-zero electric potential drop between the electrodes is responsible for the process. In AGN jet or stellar jet cases, the injection process could also be accompanied by electric potential drop in the radial direction as a result of interaction among the central object, wind, magnetic field, and the accretion disk dynamics, such as differential rotation of the disk and corona. However, the artificial injection of a purely toroidal field should produce mathematically equivalent magnetic structure. This injection is also consistent with the asymptotic X-winds solution by Shu et al. (1995) and Shang et al. (2006).

\subsubsection{Jet Collimation as a Result of Helicity/Energy Injection}

To illustrate how injected toroidal magnetic field impacts the system, we consider a "virtual magnetic field" configuration composed by $\mathbf{B}_{\text {pol }}$ (defined in Equations (3)-(5)) and $\mathbf{B}_{\text {tor }}$ (defined in Equation (13)). The Lorentz force

$$
\begin{aligned}
\mathbf{F} & \equiv \mathbf{J} \times \mathbf{B}=\left(\mathbf{J}_{\text {pol }}+\mathbf{J}_{\text {tor }}\right) \times\left(\mathbf{B}_{\text {pol }}+\mathbf{B}_{\text {tor }}\right) \\
\mathbf{F}_{\text {pol }} & =\mathbf{J}_{\text {pol }} \times \mathbf{B}_{\text {tor }}+\mathbf{J}_{\text {tor }} \times \mathbf{B}_{\text {pol }} \\
\mathbf{F}_{\text {tor }} & =\mathbf{J}_{\text {pol }} \times \mathbf{B}_{\text {pol }}
\end{aligned}
$$

has both poloidal and toroidal components.

We first examine the toroidal component of the Lorentz force, or, the twist force. The first component of $\mathbf{J}_{\text {pol }}$ in Equation (14) is parallel to $\mathbf{B}_{\text {pol }}$, hence only the second term contributes to the twist, namely,

$$
\begin{aligned}
\mathbf{F}_{\text {tor }}= & \frac{\alpha_{t} \Psi_{\mathrm{pol}} A z e^{-A z^{2}}}{\pi r} \hat{r} \times B_{z} \hat{z}=-2 \alpha_{t} \alpha_{p}^{2} A \frac{r z}{\left(l^{2}+a_{0}^{2}\right)^{4}} e^{-2 l^{2}-A z^{2}} \\
& \times\left[2\left(1-r^{2}\right)\left(l^{2}+a_{0}^{2}\right)-3 r^{2}\right] \hat{\theta} .
\end{aligned}
$$

For small radius, the twist force scales as $F_{\text {tor }} / r \sim$ $z e^{-(A+2) z^{2}} /\left(z^{2}+a_{0}^{2}\right)^{3}$. The twist force is strongest at $z=0.166 \Rightarrow$ $3 \mathrm{~cm}$ and weak for very small $z$ and large $z$. In the simulation, $F_{\text {tor }}$ twists the plasma differently at different radii and height, and hence contributes to $E_{r}$ by increasing $v_{\theta} B_{z}$ negatively. This electric field is equivalent to the voltage across the inner cathode and outer anode in the experiment.

In the poloidal component of the Lorentz force, the $\mathbf{J}_{\text {tor }} \times \mathbf{B}_{\text {pol }}$ term is the hoop force that expands the system resulting from the poloidal magnetic field; while $\mathbf{J}_{\text {pol }} \times \mathbf{B}_{\text {tor }}$ is the pinch force and is caused solely by the toroidal magnetic field. Insertion of Equations (4), (5), (7), (14), and (13) into Equation (16) yields

$$
\begin{aligned}
F_{r}= & -\frac{\alpha_{p}^{2} r e^{-2 l^{2}}}{\left(l^{2}+a_{0}^{2}\right)^{6}}\left[2\left(1-r^{2}\right)\left(l^{2}+a_{0}^{2}\right)-3 r^{2}\right] \\
& \cdot\left[g(l)+\alpha_{t}^{2} e^{-2 A z^{2}}\left(l^{2}+a_{0}^{2}\right)^{2}\right] \\
F_{z}= & \frac{\alpha_{p}^{2} r^{2} z e^{-2 l^{2}}}{\left(l^{2}+a_{0}^{2}\right)^{6}}\left[\left(3+2 l^{2}+2 a_{0}^{2}\right) g(l)\right. \\
& \left.+\alpha_{t}^{2} e^{-2 A z^{2}}\left(l^{2}+a_{0}^{2}\right)^{2}\left(3+4 l^{2}+4 a_{0}^{2}\right)\right] .
\end{aligned}
$$

The terms containing $g(l)$ result from the poloidal field and the terms proportional to $\alpha_{t}^{2}$ are given by the toroidal field. In the small $\alpha_{t}$ limit, the pinch applied by the toroidal field is weak, so the $g(l)$ term determines the direction of the Lorentz force. In the region of small $r$ and $l, F_{r}>0$ and $F_{z} / z<0$, showing that the plasma expands and is made more diffuse by the hoop force. The same argument is true for $l<l_{0} \approx 1$ and for finite $\alpha_{t}$ with $A z^{2} \gg 1$. In the cases where $\alpha_{t}$ is sufficiently large, i.e., the pinch due to the poloidal current/toroidal field overcomes the hoop force, $F_{r}<0$ and $F_{z} / z>0$ for small $r$. This is where the toroidal field squeezes the plasma radially and lengthens it axially. To see this more clearly, if we ignore the poloidal field effect by dropping the terms containing $g(l)$, the radial Lorentz force is $F_{r} / r \propto e^{-2(A+1) z^{2}} /\left(l^{2}+a_{0}^{2}\right)^{3}$, which decreases rapidly along the $z$ axis. Hence, the plasma is pinched and pressurized more at small $z$ than at large $z$. The huge pressure gradient along the central axis, due to the huge gradient of collimation force, then accelerates the plasma away from the $z=0$ plane. Equivalently, the large gradient of the toroidal magnetic pressure $B_{\theta}^{2} /\left(2 \mu_{0}\right)$ in the $z$ direction is responsible for the collimation and acceleration of the plasma. 

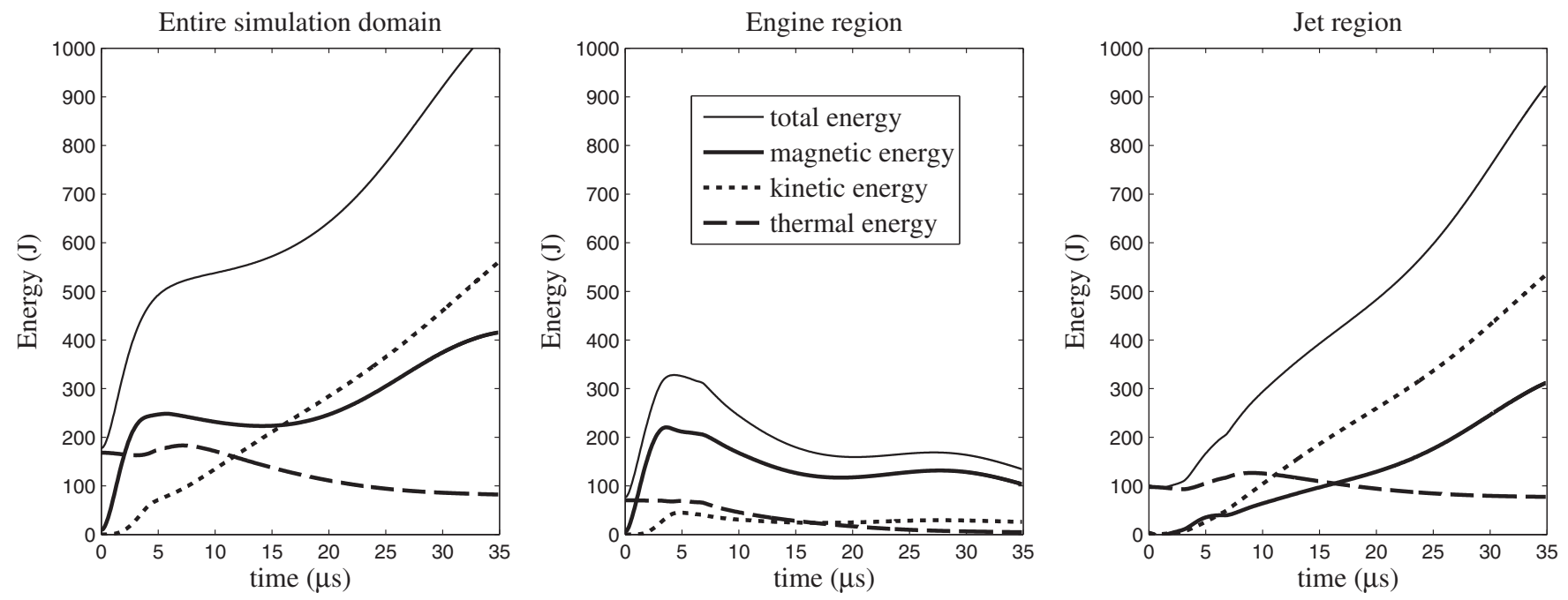

Figure 2. Evolution of different energy components in the entire simulation domain (left), engine region $|z|<z_{\text {foot }}$ (middle), and jet region $|z| \geqslant z$ foot (right).

It is important to point out that the Lorentz force is primarily poloidal. Since

$$
\frac{F_{\text {tor }}}{F_{r}}=\frac{2 \alpha_{t} A z e^{-A z^{2}}\left(l^{2}+a_{0}^{2}\right)^{2}}{g(l)+\alpha_{t}^{2} e^{-2 A z^{2}}\left(l^{2}+a_{0}^{2}\right)^{2}} \propto\left\{\begin{array}{c}
\alpha_{t} \text { for small } \alpha_{t} \\
1 / \alpha_{t} \text { for large } \alpha_{t}
\end{array},\right.
$$

$F_{r}$ is usually much stronger than $F_{\text {tor }}$.

The above analyses show that the Lorentz force tends to squeeze the plasma radially and accelerate it axially with the presence of $\mathbf{B}_{\text {tor }}$. However, in the simulation, only $\mathbf{B}_{\mathrm{pol}}$ is initially imposed as the bias poloidal field. The toroidal field is continuously injected into the system at small $z$. Meanwhile, the existing poloidal and toroidal magnetic field configuration is continuously deformed together with the plasma. Equations (17) and (18) are not exact expressions of the Lorentz force experienced by the plasma. However, Equations (17) and (18) nevertheless gives a semi-quantitative description of how injected toroidal field affects the plasma.

In summary, we have established both the initial condition and the continuous injection condition for simulating the Caltech plasma jet experiment. Only a poloidal field and a dense plasma distributed roughly parallel to the field lines are imposed initially. As the plasma starts to evolve, although the hoop force by the initial toroidal current tries to expand the plasma radially, the injected toroidal magnetic field (poloidal current) applies Lorentz force that overcomes the poloidal field pressure, and squeezes the plasma radially and lengthens it axially to form a jet in both the $+\hat{z}$ and $-\hat{z}$ directions. We only consider the $+z$ part as the $-z$ part is a mirror image.

\section{SIMULATION RESULTS}

In this section, we present some typical simulation results and compare them to the experimental results.

\subsection{A Typical Argon Jet Simulation}

First, we show a typical argon plasma jet simulation $(\mu=$ 40). The initial poloidal flux factor is selected as $\alpha_{p}=10$ corresponding to a $1.59 \mathrm{mWb}$ poloidal flux with maximum $B_{z}$ strength of $0.165 \mathrm{~T}$ at the origin. The initial mass distribution is given by Equation (9) with $\delta=40$ and $n_{\text {init, } 0}=4000$, corresponding to a maximum initial electron number density $2 \times 10^{22} \mathrm{~m}^{-3}$.
The dimensionless injection coefficient is

$$
\gamma_{b}(t) \alpha_{t}=1000 e^{-30 t}+150 e^{-20(t-0.5)^{2}}
$$

for $0 \leqslant t \leqslant 0.6=35 \mu \mathrm{s}$, which contains a short exponential decay and then a long-duration Gaussian profile. This corresponds to the fast power input by the main capacitor and then the long-duration power input by the PFN in the experiment. This injection rate is obtained based on the experiment current characteristics. In the experiment, the main capacitor gives rise to a plasma poloidal current at a rate of $\approx 150 \mathrm{kA} / 3 \mu \mathrm{s} \times(\pi / 2) \sim 10^{2} \mathrm{kA} / \mu \mathrm{s}$. The PFN supplies current $60-80 \mathrm{kA}$ with a rise time of $\sim 10 \mu \mathrm{s}$, giving a current injection rate $\sim 10 \mathrm{kA} / \mu \mathrm{s}$. With $\alpha_{p}=10$, Equation (15) indicates a dimensionless injection rate $\gamma_{b} \alpha_{t} \sim 10^{3}$ for the main capacitor and $\sim 10^{2}$ for the PFN.

The localization factor is $A=9$ so the engine region extends up to $z_{\text {foot }}=0.307 \Rightarrow 5.5 \mathrm{~cm}$. The initial plasma temperature is uniformly $T_{i}=T_{e}=2 \mathrm{eV}$, and the plasma remains $100 \%$ singly ionized through the simulation.

\subsubsection{Global Energy Analysis}

First, we examine the overall global energetics of the jet. The kinetic energy, magnetic energy and thermal energy in different regions are calculated by integrating dimensional quantities $\rho v^{2} / 2, e_{B}=B^{2} /\left(2 \mu_{0}\right)$ and $P_{g} /(\gamma-1)$ over the volume of interest for comparison with experiment. The evolution of these various types of energy are plotted in Figure 2.

The simulation starts with a finite thermal energy and a small magnetic energy from the initial poloidal magnetic field. During the first $5 \mu \mathrm{s}$, the toroidal field is injected into the engine region at a very fast rate, leading to a rapid rise in total magnetic energy. Meanwhile, the injected toroidal field continuously applies a Lorentz force to the plasma, converting magnetic energy into kinetic energy. At $5 \mu$ s, this energy conversion rate exceeds the declining toroidal field injection rate, and the magnetic energy of the entire simulation domain begins to drop. This dropping trend is terminated by the second fast injection occurring at later time. At $10 \mu \mathrm{s}$, the relative amounts of magnetic and kinetic energy in the engine region reach a quasi-equilibrium state where magnetic energy dominates and remains roughly constant. However, the magnetic and kinetic energy in the jet region continue growing at constant rates. Therefore, magnetic 

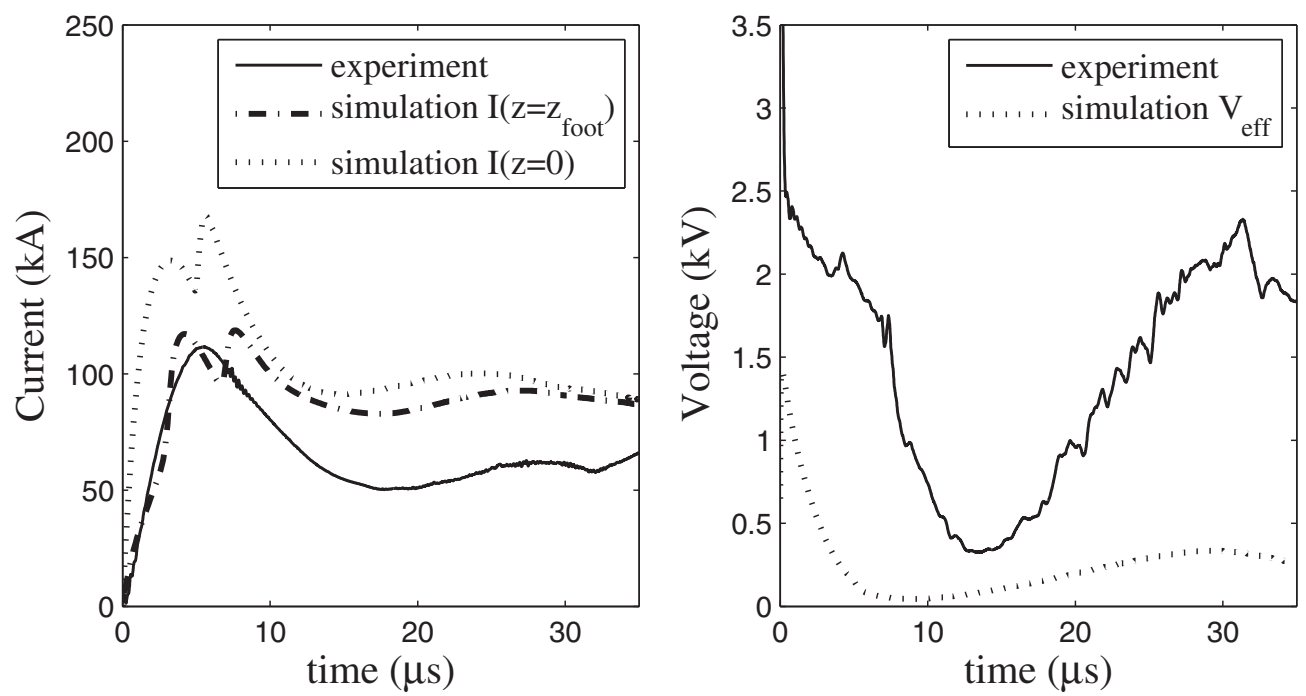

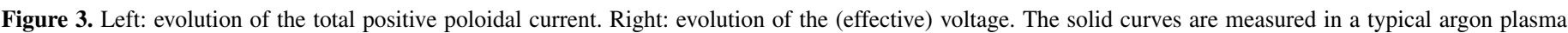

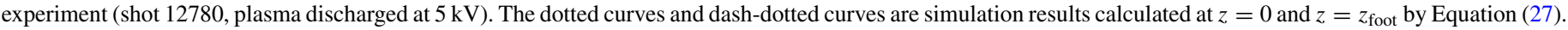

energy injected in the engine region is effectively transferred to the jet region because the energy in the engine region stays saturated. The energy partition and evolution are consistent with estimation for the experiment(see Kumar 2009, Chapter 3).

The thermal energy is insignificant compared to the magnetic and kinetic energies. The thermal energy has a small rise in early time due to the adiabatic heating from the collimation, and then slowly decreases because of the mass loss at the domain boundaries. Heating during the jet evolution is in general also not important in the experiment.

In Section 3.3.2, we showed that the jet is accelerated by the plasma pressure gradient along the central axis. This pressure gradient is caused by the non-uniform toroidal field pinching. In the jet region, the rate of increase of kinetic energy greatly exceeds the decrease of the thermal energy. Therefore it is confirmed that the jet gains kinetic energy ultimately from magnetic energy, not from thermal energy, i.e., the jet is magnetically driven.

The total power input into the system is given by

$$
P_{\mathrm{tot}} \equiv \iiint\left(\dot{e}_{B}+\dot{e}_{K}+\dot{e}_{T}\right) d V,
$$

where $e_{B}, e_{K}$ and $e_{T}$ are the magnetic, kinetic, and thermal energy density.

If we ignore the energy loss due to the outflow mass at the solving domain boundaries, the energy conservation law states that the rate of change of total energy equals the energy injection rate associated with toroidal field injection, i.e.,

$$
P_{\mathrm{tot}}=P_{\mathrm{inj}} \equiv \iiint \dot{e}_{\mathrm{inj}} d V \quad \dot{e}_{\mathrm{inj}} \equiv \gamma_{b}(t) \mathbf{B}_{\mathrm{tor}} \cdot \mathbf{B} .
$$

According to the analysis in Section 3.3.1, the power injection mainly occurs in the engine region, i.e.,

$$
P_{\text {inj }} \approx \iiint_{|z|<z_{\text {foot }}} \dot{e}_{\text {inj }} d V \equiv P_{\text {inj,engine }} .
$$

Due to energy saturation in the engine region, there is also

$$
P_{\mathrm{tot}} \approx \iiint_{|z|>\geqslant z_{\mathrm{foot}}}\left(\dot{e}_{B}+\dot{e}_{K}+\dot{e}_{T}\right) d V \equiv P_{\text {jet }} \quad \text { at large } t .
$$

Therefore,

$$
P_{\text {inj,engine }} \approx P_{\text {jet }} \text { at large } t .
$$

This shows that the power input at the jet base is mainly used to accelerate the jet, and not for heating.

An effective voltage at the $z=0$ plane can be defined as

$$
V_{\mathrm{eff}} \equiv \frac{P_{\mathrm{tot}}}{I(z=0)} \quad I(z)=\iint_{J_{z}>0} J_{z} d x d y,
$$

where $I(z)$ is the total positive poloidal current through the plane $z$.

Figure 3 shows that the poloidal current in the simulation is in good agreement with the experimental measurement. At $t \leqslant 3 \mu \mathrm{s}$, the current at $z=z_{\text {foot }}$ is less than $30 \%$ of the current at the $z=0$ plane. This is because most of the toroidal injection occurs within the engine region. However, for $t>5 \mu \mathrm{s}$, the current entering the jet region is comparable with the total current in the system, indicating that the engine region is injecting toroidal flux into the jet region.

It is difficult in the experiment to measure the voltage across the plasma precisely because the impedance of the plasma is very low. The voltage measurement given by the solid curve in Figure 3 contains the plasma voltage drop as well as voltage drops on the cables and connectors. The effective voltage in the simulation is expected to be comparable to, but lower than, the experiment measurement, as is generally the case in Figure 3.

The global energy and electric characteristics comparison between the simulation and experiment confirm that the simulation captures the essential features. The jet is MHD-driven and gains kinetic energy from magnetic energy. In the following sections, we discuss the detailed process of jet collimation and propagation and various properties of the jet.

\subsubsection{Jet Collimation and Propagation}

According to the analysis in Section 3 the $A=9$ localized toroidal field injection, quantified by Equation (21), will generate a pinch force that collimates the plasma near the $z=0$ plane. Meanwhile, the plasma pressure gradient along the axis, caused by the $z$ gradient of collimation force on the jet surfaces, will accelerate the plasma away from the $z=0$ plane. 
$\log _{10}\left(\mathrm{n} \cdot \mathrm{m}^{3}\right)$ color map with poloidal flux contours
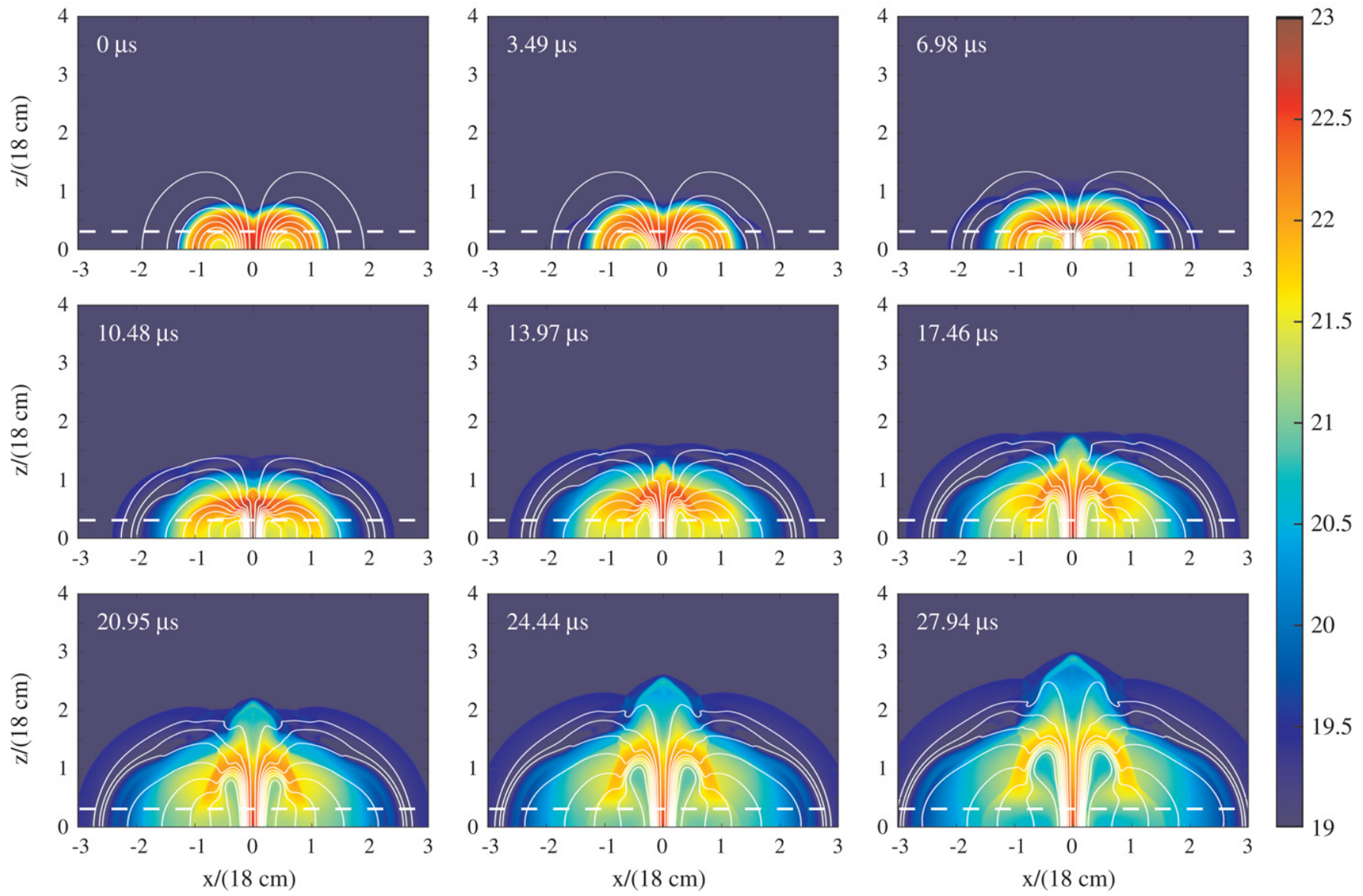

Figure 4. Evolution of the density distribution (color map) and azimuthally averaged poloidal flux surfaces (white contours) in the $x z$ plane $(z>0)$ from $t=0$ to 0.48 with 0.06 interframe time, corresponding to dimensional time from 0 to $27.94 \mu$ s with interframe time $3.49 \mu$ s. The color represents the common logarithm of the total particle number density $n=n_{e}+n_{i}$ in $\mathrm{m}^{-3}$. Each frame contains 13 evenly spaced flux contours from $0.05 \mathrm{mWb}$ to $1.45 \mathrm{mWb}$ every $0.2 \mathrm{mWb}$. The white horizontal dashed lines in each frames mark the position of $z_{\mathrm{foot}}=0.307 \Rightarrow 5.5 \mathrm{~cm}$.

(A color version of this figure is available in the online journal.)

The evolution of the plasma is given in Figure 4 which presents the time sequence of plasma density in the $x z$ ( $r z)$ plane overlaid by azimuthally averaged poloidal magnetic field contours. Figure 4 shows that plasma with frozen-in poloidal field is pinched radially and lengthened axially. Starting from a torus structure around the origin, the plasma eventually forms a dense collimated jet with a radius $r \simeq 0.2 \Rightarrow 3.6 \mathrm{~cm}$ (at $z=0$ ) and height $h \simeq 2 \Rightarrow 36 \mathrm{~cm}$ at $\sim 30 \mu \mathrm{s}$. The radius-length ratio of the plasma decreases from $\approx 1: 1$ to $\approx 1: 10$. Consequentially, a more than five times amplification of density and poloidal field is observed to be associated with the collimation process in the simulation, consistent with the experimental measurement by Yun et al. (2007). The jet radius $r \simeq 0.2$ at $z=0$ in the simulation is found where plasma density drops below $5 \%$ of the central density $\rho(r=0, z)$. An unmagnetized hydrodynamic shock bounding the global structure forms in the numerical simulation and propagates outward, as a result of supersonic jet flow propagating into the finite pressure background; this shock is not observed in the experiment because of the lack of background plasma. Here, we define the jet head as the leading edge of dense magnetized plasma along the central axis. This leading edge corresponds to the top of the $T$-shaped shell in Figure 4 (from $z=0$ to $z \sim 1.8$ at $27.94 \mu \mathrm{s}$, see also in Figure 5). The jet head is the point where all the poloidal flux bends and returns back to the mid-plane. In front of the jet head, plasma is essentially unmagnetized and the density drops from $\sim 10^{22} \mathrm{~m}^{-3}$ to $<10^{20} \mathrm{~m}^{-3}$. Therefore, the hydro shock and its downstream region from the $T$-shell to the shock front are not considered as part of the jet, but rather the termination of the entire global structure. Figure 4 also shows that the entire plasma structure remains axisymmetric in the simulation.

The high speed images of the experiment plasma jets shown in Figure 1 are integration of plasma atomic line emission along the line of sight. Generally atomic line emission is proportional to the square of density. Therefore, we calculate the lineof-sight integration of density squared of the simulation jet and plot the equivalent "emission" images in Figure 5, along with five experimental plasma images. The plasma is optically thin. Figure 5 shows that simulation and experimental jets have similar radius, length/velocity, brightness variation, and the relatively flat and bright plasma at jet head, a $T$-shaped structure. This $T$-shaped structure is a signature of return flux (also see the structure at the top of the jet in Figure 4). Due to the lack of any background pressure, the experimental jet has a much flatter return flux structure, compared to the $T$ shaped structure shown in simulation images at later times. This structure is much dimmer in Figure 1 because for those figures the camera was not placed perpendicular to the jet so the line of sight does not lie entirely in the $T$-shell structure. Note that the experimental jet starts to kink at $20 \mu$ s but the jet still 

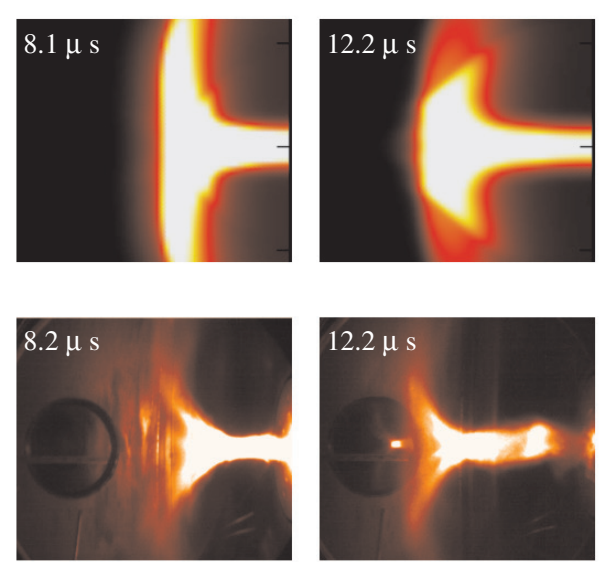

simulation
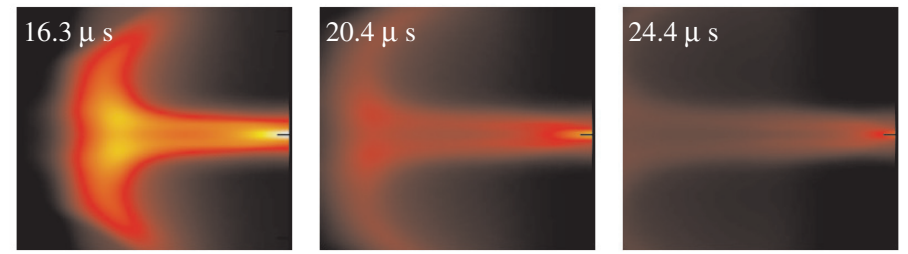

experiment
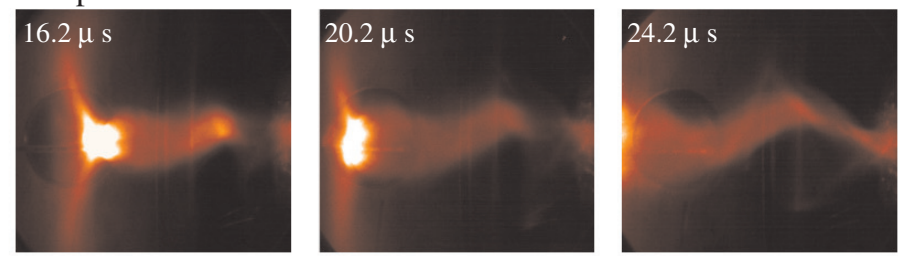

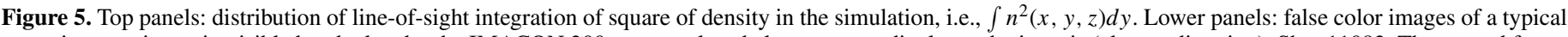

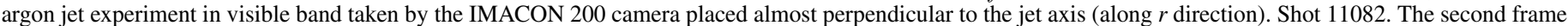

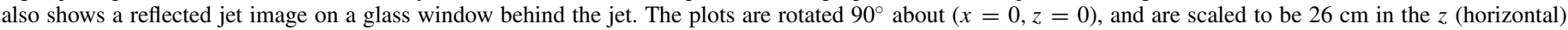
direction by $22 \mathrm{~cm}$ in the $x$ (vertical) direction. The respective color tables for both the simulation and experimental images do not change with time.

(A color version of this figure is available in the online journal.)

propagates in a similar manner and remains attached to the center electrode.

Although the localized toroidal field (poloidal current) injection is confined to the engine region $\left(|z|<z_{\text {foot }}\right.$, below the dashed lines in Figure 4), the plasma nevertheless collimates in the jet region. This is because the poloidal current, pre-injected in the engine region, propagates into the jet region along with the plasma motion and so provides a pinch force to collimate the plasma there (Figure 3, also see Figure 9 in the next section). Hence, the toroidal field injection actually occurs in both the engine region and jet region. The injection in the engine region is realized artificially by Equation (1d), a non-ideal process; the injection in the jet region is achieved through the $z=z_{\text {foot }}$ plane associated with the plasma dynamics.

The detailed axial profile of the collimated jet is given in Figure 6, which plots density, kinetic, and magnetic profiles along the central $z$ axis spanning from $11.6 \mu$ s to $30.2 \mu \mathrm{s}$. Although the experimental jet already undergoes a kink instability as early as $\sim 20 \mu \mathrm{s}$, the simulation results at late times can still be used to study the expansion of the length of the axis of the kinked experimental jet according to Figure 5 .

The left four panels (A-D) in Figure 6 show the evolution of jet's kinetic properties. The number density plots (panels (A) and (B)) show that mass is rearranged to become more elongated and more evenly distributed along the jet. Since the total mass is conserved in the solving domain, consequentially, the density or column density decreases along the jet body when the jet gets longer. Panels (C) and (D) show the axial velocity and kinetic energy are gradually developed along the jet. The plasma axial velocity decreases in the lab frame because of the jet elongation. In fact, panel (C) indicates that the axial velocity approximately follows a self-similar profile $v_{z}(t, z) \propto z / t$. Detailed calculation finds that $t v_{z} / z$ approaches 1 for $z>z_{\text {foot }}$ at later time, i.e., $v_{z} \rightarrow z / t$. Therefore, the acceleration in the frame of jet is $d v_{z} / d t=\partial_{t} v_{z}+v_{z} \partial_{z} v_{z}=0$. This means that the jet has reached a dynamic steady state and the entire jet is elongating as a whole. However, it is crucial to point out that the $v_{z} \propto z / t$ behavior is only true at later times, when the injection rate varies very slowly. At early times when the injection rate has a large variation, the jet velocity profile is expected to be very different from self-similar behavior, with density accumulation/ attenuation in some parts of the jet and even internal shocks. At the jet head, plasma flow slows down in the moving frame of plasma, density accumulation always occurs (see panel (B)), which is also observed in experiments (Yun \& Bellan 2010). This accumulation can be regarded as an indicator of jet head, e.g., $z \approx 16 \mathrm{~cm}$ at $t=16.3 \mu \mathrm{s}$ and $z \approx 28 \mathrm{~cm}$ at $t=25.6 \mu \mathrm{s}$. This gives a jet speed of $v_{z} \approx 13 \mathrm{~km} \mathrm{~s}^{-1}$, consistent with the experiment (Figure 5).

The jet speed is faster than the background plasma sound speed $c_{s}=3.1 \mathrm{~km} \mathrm{~s}^{-1}$. The supersonic jet flow is expected to excite a hydro shock with speed $v_{s}=[(3 \gamma-1) /(6 \gamma-$ $\left.4)+\sqrt{(3 \gamma-1)^{2} /(6 \gamma-4)^{2}+c_{s}^{2} / v_{z}^{2}}\right] \cdot v_{z} \approx 18 \mathrm{~km} \mathrm{~s}^{-1}$ where the adiabatic constant is $\gamma=5 / 3$ (Kulsrud 2005). This is consistent with the simulation results in panel (C). Under the strong shock approximation $v_{z} \gg c_{s}$, the shock speed is $v_{s} \approx[(3 \gamma-1) /(3 \gamma-2)] \cdot v_{z}$. In the experiment, although a hydro shock is also expected, it is not feasible to measure it because the background density is too low. Moser (2012) and Moser \& Bellan (2012b) had a $v_{z} \approx 16 \mathrm{~km} \mathrm{~s}^{-1}$ argon experiment jet collide with a pre-injected hydrogen neutral cloud with density $n \sim 10^{19}-10^{20} \mathrm{~m}^{-3}$, and observed a hydro shock in the cloud with a speed of $v_{s} \sim 25 \mathrm{~km} \mathrm{~s}^{-1}$. This satisfied the strong shock solution with $\gamma=7 / 5$ for neutral diatomic gas.

Yun \& Bellan (2010, Figures 15 and 17) measure the density and velocity profiles of a typical nitrogen jet using Stark broadening and Doppler effect. It is found that the experimental jet has a typical density $(0.5-1.0) \times 10^{23} \mathrm{~m}^{-3}$, and the density profiles behave very similarly to the argon simulation jet in aspects like mass distribution, time-dependent profile evolution, and density accumulation at the jet head, especially for the column number density (Figure 6 panel (B)). The velocity profiles of the experiment nitrogen jet also show similar trends as Figure 6 panel (C), e.g., velocity behind the jet head slows down in lab frame and the jet head travels at a roughly constant speed. In the experiment, because there is negligible background density, the measurable plasma velocity reaches zero at the jet head. In the simulation, however, the axial velocity profiles are terminated by the hydro shock in front of the jet head. Yun \& Bellan (2010) show a smaller density decrease of the jet in the experiment than in the simulation, due to the continuous mass injection into the plasma through the gas feeding holes on the electrodes (Stenson \& Bellan 2012). Continuous mass injection is not included in the simulation in order to reduce complexity. 
particle number density along $\mathrm{r}=0$
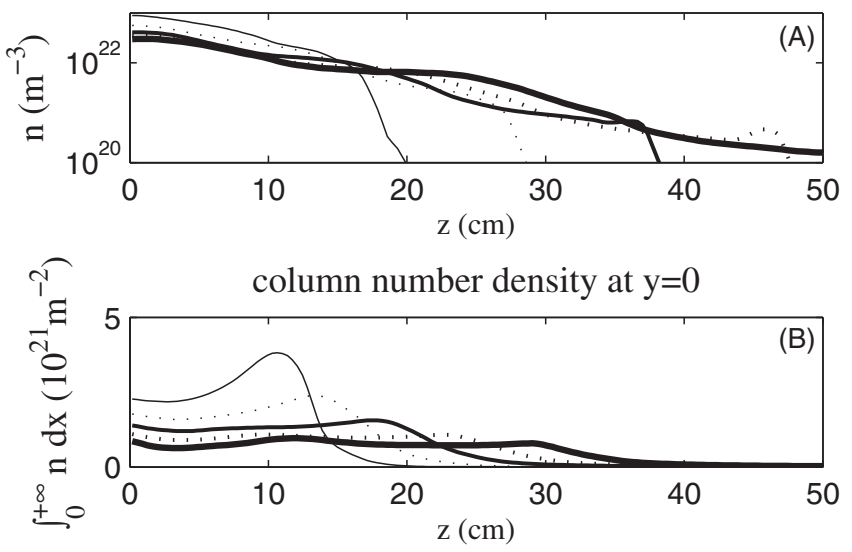

Vz along $r=0$

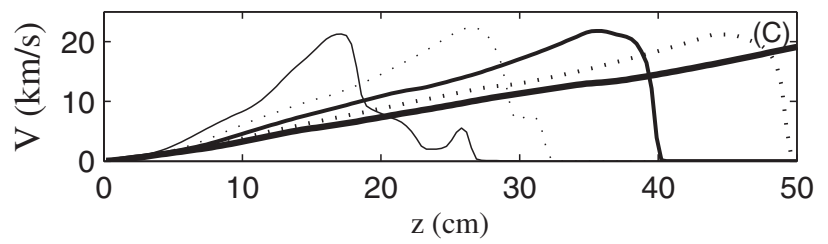

Kinetic energy density along $r=0$

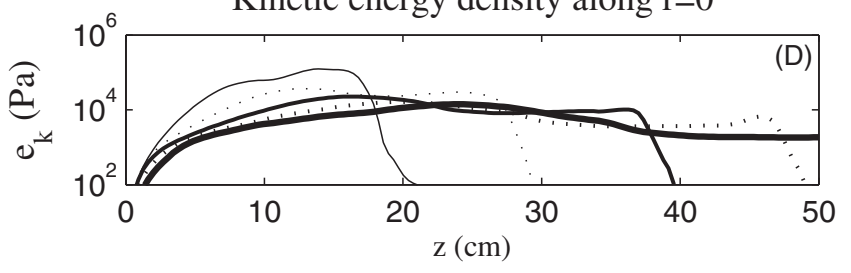

Bz along $\mathrm{r}=0$
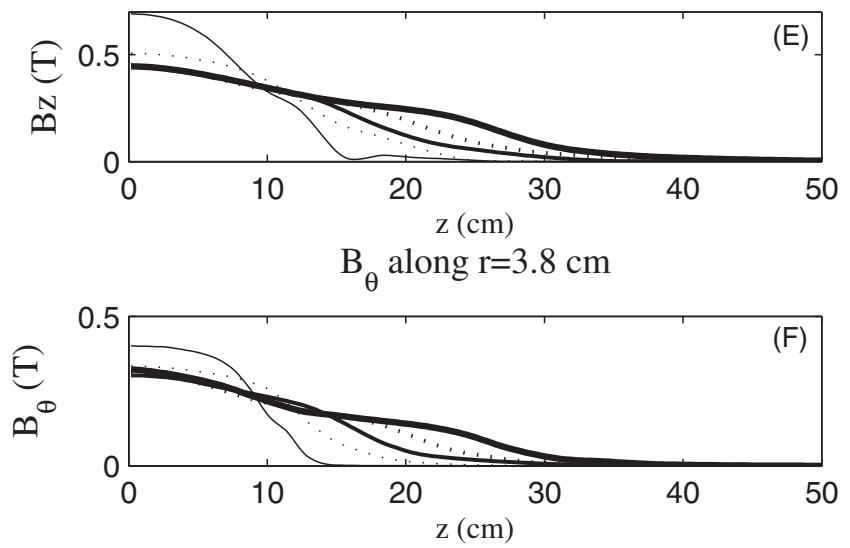

Total poloidal current at different height
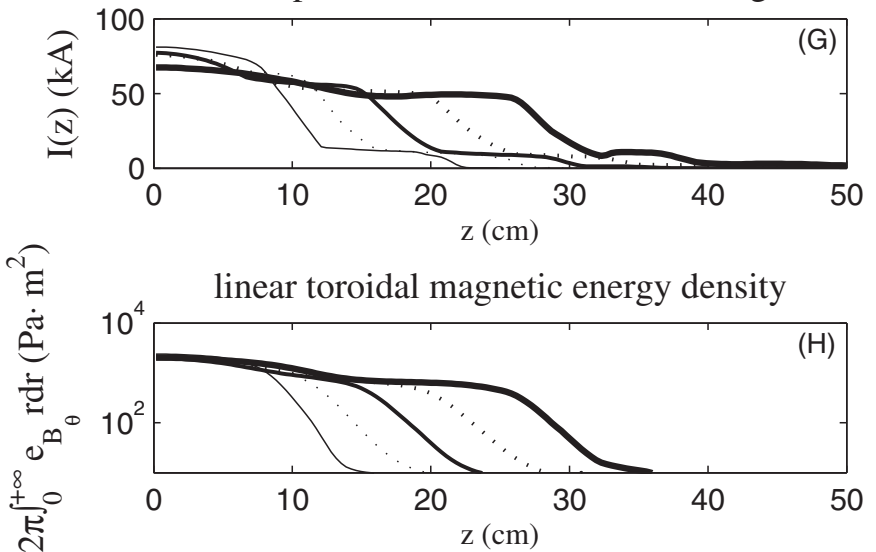

$-11.6 \mu \mathrm{s} \cdots \cdots \cdot 16.3 \mu \mathrm{s}-20.9 \mu \mathrm{s} \cdots \cdots 2.6 \mu \mathrm{s}-30.2 \mu \mathrm{s}$

Figure 6. Particle number density along central axis $n(r=0, z)$ (panel (A)), column particle number density $\int n d x$ along $y=0$ (panel (B)), axial velocity $v_{z}$ along $r=0$ (panel (C)), axial kinetic energy density $e_{k}=\rho v_{z}^{2} / 2$ along $r=0$ (panel (D)), axial magnetic field $B_{z}$ along $r=0$ (panel (E)), toroidal magnetic field $B_{\theta}(r, z)$ at $r=0.21(3.8 \mathrm{~cm})($ panel $(\mathrm{F}))$, total poloidal current $I(z) \equiv \max _{r} I(r, z)$, where $\mu_{0}(r, z)=B_{\theta}(r, z) / 2 \pi r($ panel $(\mathrm{G}))$, and total toroidal field energy at each height $\int_{0}^{\infty} e_{B_{\theta}} d \theta r d r(\operatorname{panel}(\mathrm{H}))$, where $e_{B_{\theta}}=B_{\theta}^{2} / 2 u_{0}$.

This results in a larger density attenuation in the simulation as the jet propagates (panel (A) and (B)). It is important to point out that the experimental nitrogen jets and argon jets do not have exactly the same conditions, so the discussion here on nitrogen jet, while identifying similar trends, is not quantitative.

As the jet lengthens, axial magnetic field embedded in the plasma is also stretched out, resulting in a quasi-uniform magnetic density along the jet axis. This is clearly evident by noticing the $B_{z}$ evolution in Figure 6 panel (E). At $11.6 \mu \mathrm{s}, B_{z}$ attenuates from $0.7 \mathrm{~T}$ to $0.35 \mathrm{~T}$ in $9.5 \mathrm{~cm}$, while at $30.2 \mu \mathrm{s}$ this two-fold decay occurs in a distance of $25 \mathrm{~cm} \approx 6$ jet radius. Hence the axial magnetic field is becoming more uniform. Panel (F), (G), and (H) demonstrate that toroidal magnetic field and poloidal current propagate along the jet body and reach the same distance as does the plasma density, despite the fact that toroidal field/poloidal current is injected in the engine region at small $z$. The jet is thus still being collimated by the toroidal field/poloidal current even though the jet is already far from the engine region. The total positive poloidal current (panel $(\mathrm{G})$ ) and total toroidal magnetic energy density (panel $(\mathrm{H})$ ) become quite uniform along the jet in later time. Panel (G) also clearly indicates the jet head location, where all poloidal current turns back and results in a sharp decrease in total positive poloidal current at the jet head. The locations of this sharp decrease is consistent with the location of density accumulation shown in panel (B).

According to Figure 6 here and Figure 17 in Yun \& Bellan (2010), there is no distinct jet head in either simulation or experiment. After the main jet body, plasma density and other characteristics, such as poloidal flux and current, take significant distance to reach zero. The reason is again the lack of background pressure. In the jet-neutral cloud collision experiment (Moser 2012; Moser \& Bellan 2012b), a sharper jet head with significant amplified density and magnetic field is observed.

Although panels (E) and (F) show that $B_{z}$ along the axis remains comparable with $B_{\theta}$ at the jet boundary, we will show in Section 4.1.3 that this result does not conflict with Lynden-Bell (1996), Lynden-Bell (2003), Sherwin \& Lynden-Bell (2007), or Zavala \& Taylor (2005), in which an increasing pitch angle $B_{\theta} / B_{z}$ is expected tracing magnetic field lines along the jet.

Figure 7 shows the distribution of Poynting flux $B_{\theta}^{2} v_{z}$, kinetic flux $\rho v_{z}^{3}$ and enthalpy flux $\gamma p /(\gamma-1) v_{z}$ at $t=29.1 \mu \mathrm{s}$. The figure shows that Poynting flux has successfully reached the height of jet head $z \approx 1.8$, even though the toroidal field is injected at $z<0.307$. Poynting flux is generally $2-10$ times larger than kinetic flux, and two to three orders of magnitude larger than thermal flux, showing that the jet is MHD-driven and magnetically dominated. However, at small radius where $B_{\theta}$ is 


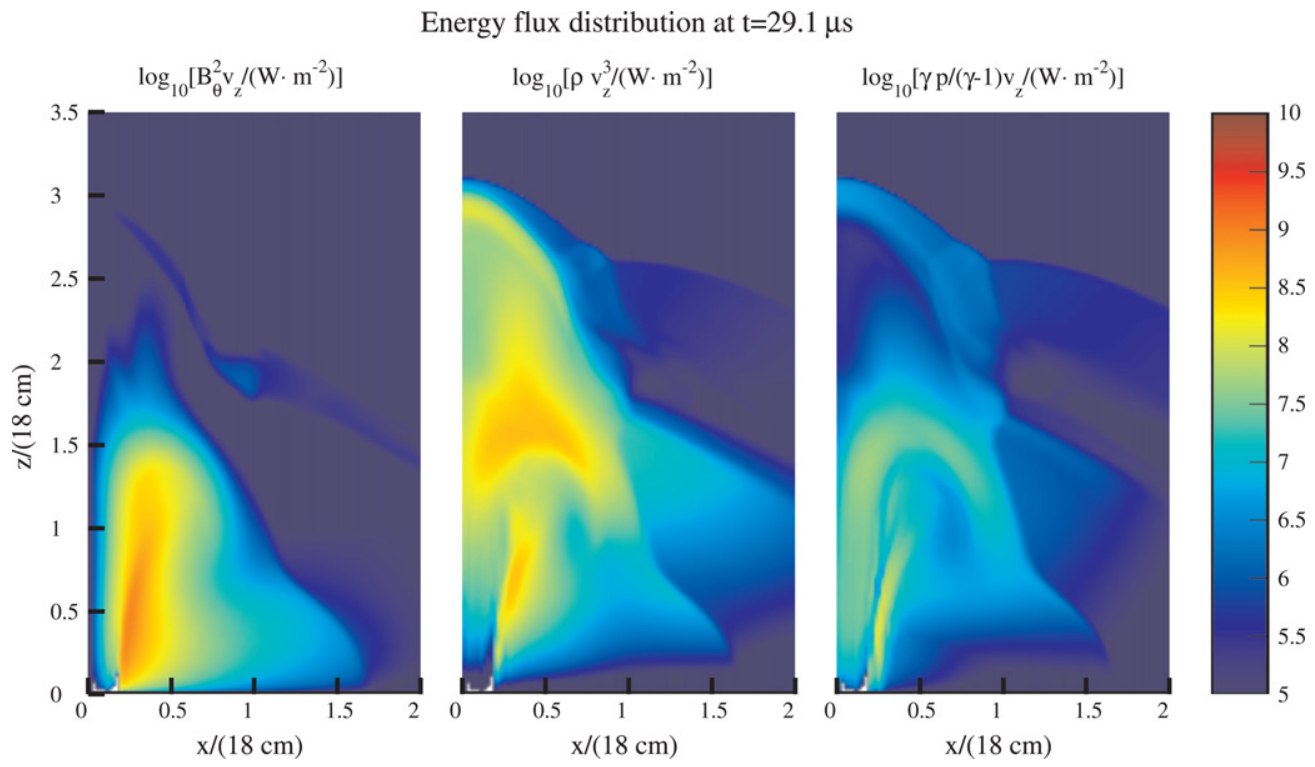

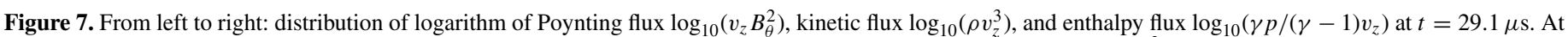
this time, the jet head is at $z \approx 1.8$ or $32 \mathrm{~m}$ and jet radius is about $r=0.2$ or $3.6 \mathrm{~cm}$. The SI unit for energy flux is W $\mathrm{m}^{-2}$.

(A color version of this figure is available in the online journal.)

small, kinetic and thermal flux are larger than Poynting flux. The hydro shock in front of the jet carries a notable amount of kinetic energy due to the fast expansion velocity.

\subsubsection{Jet Structure and the Global Magnetic Field Configuration}

We have shown that a collimated jet automatically forms in the jet region when toroidal field is injected into the engine region. We now examine the jet structure in the jet region.

Figure 8 plots the radial profiles of the plasma density, pressure, velocity, and magnetic field at $z=1.14 \Rightarrow 20.5 \mathrm{~cm}$ $\left(15 \mathrm{~cm}\right.$ above the $z_{\text {foot }}$ plane) at different times. At $5.8 \mu \mathrm{s}$, according to Figure 4, a collimated jet structure has not yet formed, and the injection in the engine region has caused little impact at $z=20.5 \mathrm{~cm}$. As expected, the left three panels of Figure 8 reveal a low density $\left(\sim 10^{19} \mathrm{~m}^{-3}\right)$, low velocity and very weakly magnetized plasma structure. (Note that the vertical scales for $5.82 \mu \mathrm{s}$ and $29.1 \mu \mathrm{s}$ are different in Figure 8). However, the negative radial velocity between 1 and $10 \mathrm{~cm}$ shows that the collimation has already started at this time. At $29.1 \mu \mathrm{s}$, a collimated jet in steady-state is expected at $z=20.5 \mathrm{~cm}$ because the jet head has travelled beyond $20.5 \mathrm{~cm}$ according to Figure 4 . The right three panels of Figure 8 show that the entire radial profile can be divided into three regions from small to large radii, namely the central column (jet, region $A$ ), the diffuse pinch region (region $B$ ), and the return flux region (region $C$; see also discussions of these structures in Nakamura et al. 2006 and Colgate et al. 2014).

Central Column. For $r \lesssim 4-5 \mathrm{~cm}$, the central jet is characterized by a $\sim 10^{22} \mathrm{~m}^{-3}$ high density, a $\sim 10 \mathrm{~km} \mathrm{~s}^{-1}$ quasi-uniform axial velocity and a $\sim 0.24 \mathrm{~T}$ axial magnetic field. The radial velocity is zero, indicating that collimation is complete and a radially balanced $z$-pinch configuration is maintained. The toroidal magnetic field gradually increases from $r=0$ to $r \approx 5 \mathrm{~cm}$ at a roughly constant slope, suggesting that the central jet is filled by a roughly uniform current $J_{z}$. The zero $B_{r}$ additionally demonstrates that the magnetic field is well confined inside the jet. At the jet boundary, density, pressure, axial magnetic field, and current density drop rapidly and connect to the diffuse pinch region. Specifically, at $r=5 \mathrm{~cm}$, the plasma density is already less than $15 \%$ of the maximal density $1.14 \times 10^{23} \mathrm{~m}^{-3}$ at $r=1.7 \mathrm{~cm}$. The density dip at $r=0$ results from the initial torus-shaped mass distribution.

Diffuse Pinch Region. For $5 \mathrm{~cm} \lesssim r \lesssim 12 \mathrm{~cm}$, there is a relatively large region filled by low density plasma $(\sim 5 \times$ $10^{20} \mathrm{~m}^{-3}$ ) surrounding the central dense jet. The toroidal magnetic field $B_{\theta}$ scales as $r^{-0.96} \approx 1 / r$ in this region, showing that the poloidal current is almost zero. Detailed calculation shows that $87 \%$ of total axial current $I_{Z}$ flows inside the central column $r<5 \mathrm{~cm}$, and another $13 \%$ of $I_{Z}$ exists in the $5 \mathrm{~cm} \lesssim r \lesssim 10 \mathrm{~cm}$ region. The axial magnetic field $B_{z}$ drops to zero with a steep scaling $B_{z} \sim r^{-5.5}$ from $5 \mathrm{~cm}$ to $8 \mathrm{~cm}$, and reverses polarity at $r=8.5 \mathrm{~cm}$. The radial magnetic field $B_{r}$ is $\lesssim 10^{-2}$ times weaker than $B_{z}$ and $B_{\theta}$. This region has a relatively fast axial velocity and finite radial velocity. However, because of the low density, the kinetic energy in this region is only $15 \%$ of the toroidal magnetic energy in the same region, and is less than $10 \%$ of the central column kinetic energy. Hence, the diffuse pinch region is a toroidal magnetic field dominant region with low $J_{z}$.

Return Flux Region. Since the simulation starts with a complete global dipole magnetic field, the poloidal flux, carried by the central jet, must return to the central plane at some point. According to Figures 4 and 6, all the upward flux frozen into the dense plasma starts to return at the jet head. The return flux at $z=20.5 \mathrm{~cm}$ is found in the narrow $12 \mathrm{~cm} \lesssim r \lesssim 15 \mathrm{~cm}$ region and has a $\sim 0.04 \mathrm{~T}$ negative strength. The toroidal field sharply decays to zero in this region as well, indicating the existence of a narrow return poloidal current sheet. The Lorentz force acting on this current sheet repels this region away from the central axis at a fast speed $\left(v_{r} \approx 6 \mathrm{~km} \mathrm{~s}^{-1}\right)$, and piles up and compress plasma in $15 \mathrm{~cm} \lesssim r \lesssim 18 \mathrm{~cm}$ and forms the $T$-shell shown in Figure 4.

The return flux region transitions to the background plasma configuration through a hydrodynamic shock at $r \approx 50-60 \mathrm{~cm}$. At $t=29.2 \mu \mathrm{s}$, since the return flux region still has higher density and pressure compared to the background, the unmagnetized shock expands radially at a supersonic velocity of $v_{s} \approx 6 \mathrm{~km} \mathrm{~s}^{-1}$ (sound speed $C_{s 0}=3.1 \mathrm{~km} \mathrm{~s}^{-1}$, see Table 1). At very late 

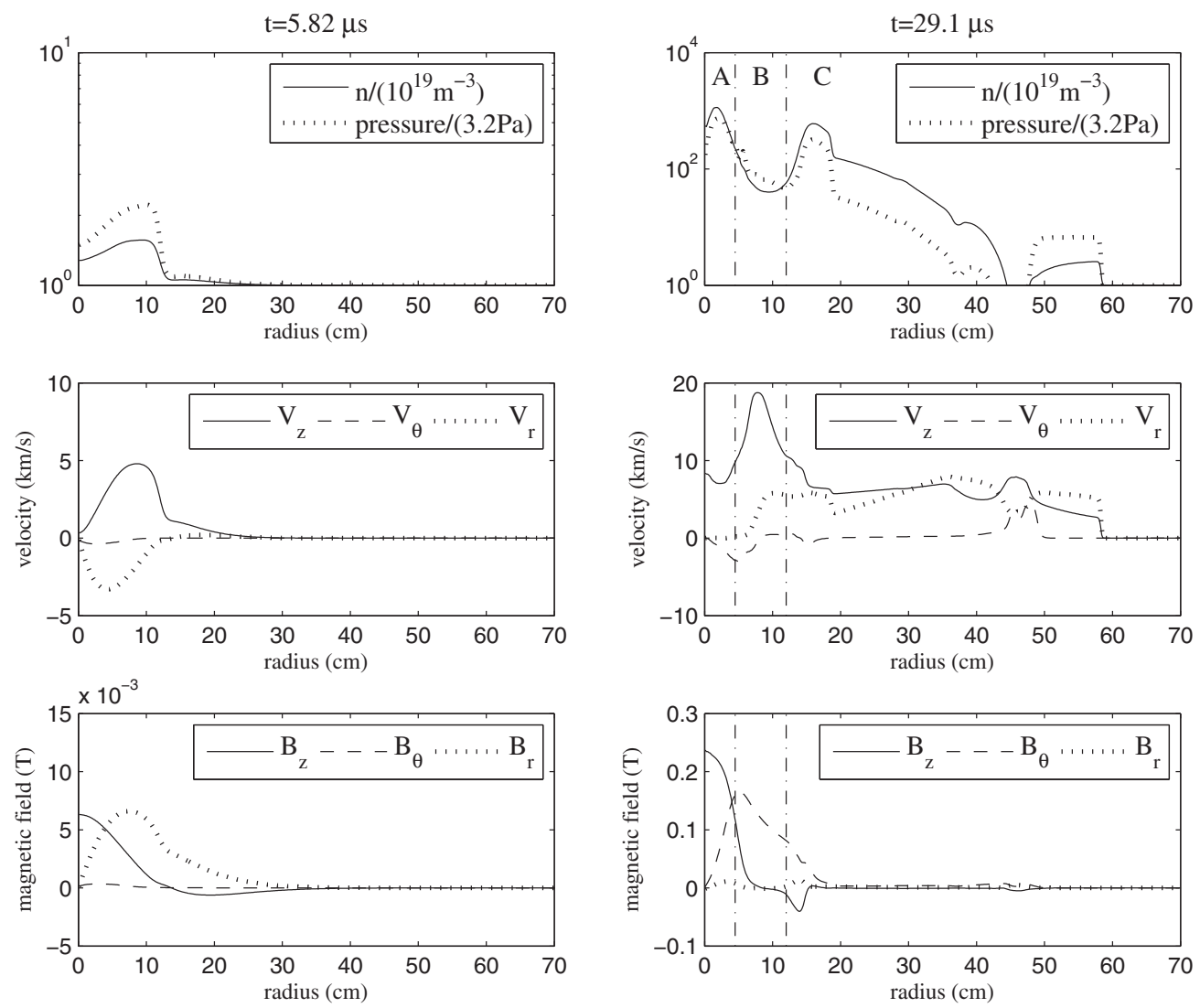

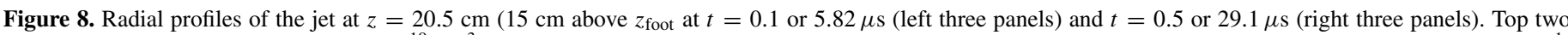

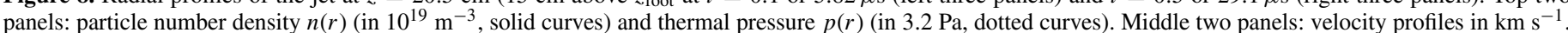

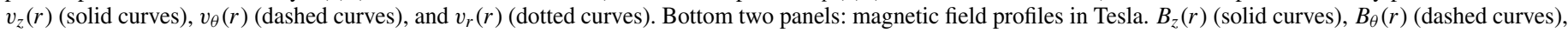

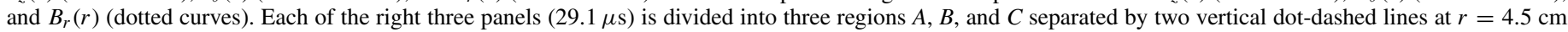
and $r=12 \mathrm{~cm}$. See Section 4.1 .3 for details.

time, when there is sufficient radial expansion, the density and pressure in the return flux region are expected to be low enough so that the expansion will become sonic. The entire jet structure is expected to transit to pressure confinement from inertial confinement (Nakamura et al. 2006).

These radial profiles of the central jet confirm that the jet is highly magnetized and is MHD-collimated. The cross-sectional view of various plasma properties in Figure 9 further validate this point. By comparing Figure 9 with Figure 4, we find that the strong poloidal field and current are both confined in the dense plasma region (central jet region and the outer boundary of the return flux region). Poloidal field, current, and toroidal field have been established from $z=0$ to $z=1.8$, same as the density and Poynting flux (Figures 4 and 7).

Figure 9 shows that the poloidal current is approximately parallel to the poloidal magnetic field in most of the region, especially in the central column, suggesting that the Lorentz force is dominantly poloidal, because the toroidal Lorentz force $\mathbf{F}_{\text {tor }}=\mathbf{J}_{\text {pol }} \times \mathbf{B}_{\text {pol }} \approx 0$. This is consistent with the analysis given by Equation (20). Detailed calculation finds that $F_{\text {tor }}$ in the simulation is generally one to three orders of magnitude smaller than $F_{\text {pol }}$. The Lorentz force distribution panel shows that $\mathbf{J} \times \mathbf{B}$ is extremely strong at the jet boundary especially at relatively low height. The Lorentz force at the jet boundary is radially inward due to the self-pinch of the poloidal current, and is responsible for the collimation. The very large gradient of this pinching force along $z$ direction $\partial_{z}\left[(\mathbf{J} \times \mathbf{B})_{r}\right]$, equivalent to the gradient of toroidal magnetic energy $\partial_{z}\left(B_{\theta}^{2}\right)_{r}$, collimates the plasma gradually from lower $z$ to higher $z$, and ultimately accelerates the plasma. This demonstrates the MHD pumping mechanism in the current-driven plasma tube proposed by Bellan (2003) and verified in the Caltech plasma jet experiment (Yun et al. 2007; Yun \& Bellan 2010; Kumar \& Bellan 2009). Figure 9 also shows that the return flux/current are expanding outward under a relatively strong Lorentz force. It is notable that at $z>0.7$ where the jet has not been fully collimated, the poloidal field is being compressed at very small radius, resulting in a radial outward Lorentz force.

The plasma $\beta$ panel shows that the central jet has a typical $\beta \approx 10^{-1.5}-10^{-1}(0.03-0.1)$, consistent with the experiment (Section 2). Hence, the jet is magnetically dominated. The $\beta$ value is even smaller in the diffuse pinch region, due to the low plasma density and relatively strong toroidal magnetic field. The hydro shock has a very high $\beta$ value since it is essentially unmagnetized.

Figure 10 compares the magnetic structure of the simulation jet with the experimental jet. The experimental measurements are obtained using the $1 \mathrm{MHz} 20$ channel MPA at $z=15 \mathrm{~cm}$ from the electrode plane (Romero-Talamás et al. 2004) in a typical argon jet experiment. The top panel shows poloidal flux contours calculated from the MPA measurement from $t=15 \mu \mathrm{s}$ to $t=25 \mu \mathrm{s}$, during which times the MPA has effectively "scanned" approximately $15 \mathrm{~cm}$ distance along the $z$ direction in the moving frame of jet, although the MPA is fixed in the lab frame. The contours show that the magnetic field lines inside the jet $(r \lesssim 5 \mathrm{~cm})$ are quite collimated. The middle 

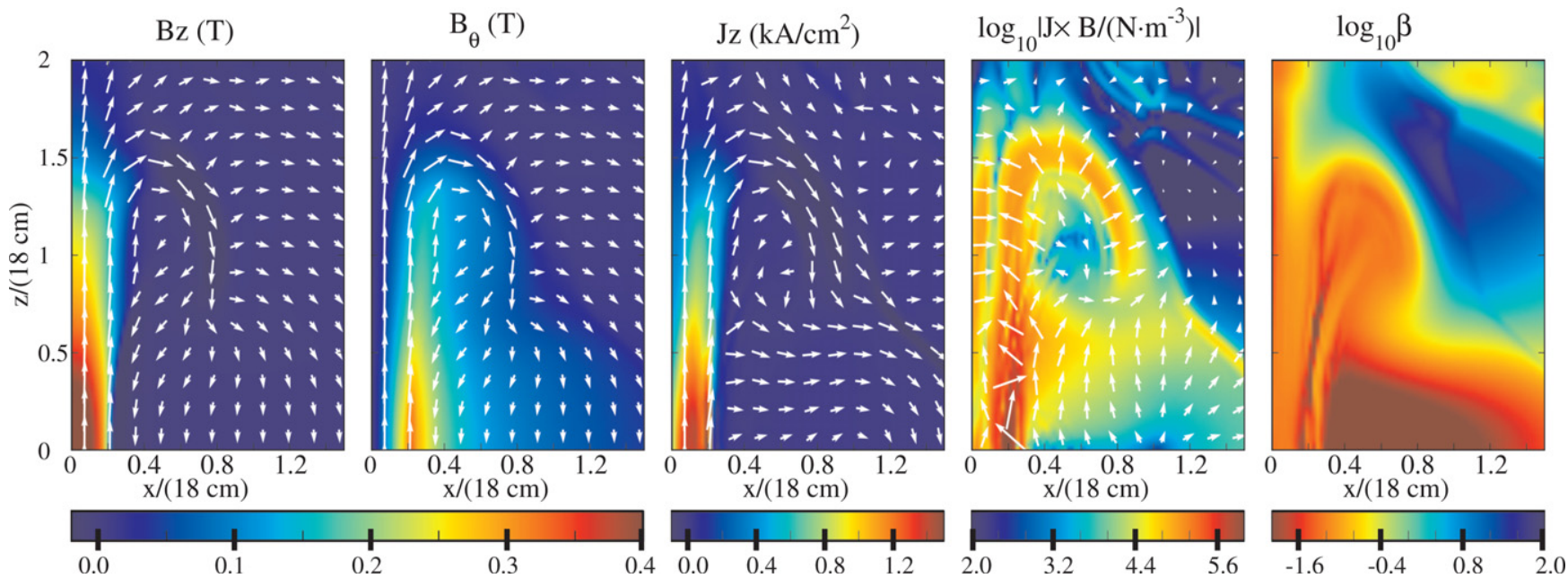

Figure 9. Cross-sectional view of plasma properties at $t=0.5$ or $29.1 \mu \mathrm{s}$. From left to right: axial magnetic field $B_{z}$ with poloidal field arrows, toroidal magnetic field $B_{\theta}$ with poloidal field arrows, axial current $J_{z}$ with poloidal current arrows, logarithm of Lorentz force density with poloidal $\mathbf{J} \times \mathbf{B}$ arrows, and logarithm of plasma $\beta$ (ratio of thermal energy density to magnetic energy density) distribution. In all panels, the length of each arrow is proportional to the one-fifth power of the corresponding quantity at the location of arrow center. For example, an arrow I with a half length of an arrow II means that the represented quantity at arrow I is only $1 / 2^{5} \sim 3 \%$ of arrow II.

(A color version of this figure is available in the online journal.)

panel plots the radial profiles of $B_{z}$ and $B_{\theta}$ at $t=22 \mu \mathrm{s}$ in the experiment. The bottom panel gives the magnetic profiles in the simulation at $z=14.4 \mathrm{~cm}+z_{\text {foot }}$ at $t=24 \mu \mathrm{s}$. In both simulation and experiment, $B_{z}$ is $\simeq 0.2 \mathrm{~T}$ at the central axis and reverses direction at $r \approx 7 \mathrm{~cm} ; B_{\theta}$ rises quasi-linearly for small $r$ and peaks at $r=5 \mathrm{~cm}$. Hence $J_{z}$ is approximately constant within the central jet. Despite the excellent agreement in the central column region, it should be noted that the return current in the experiment extends to a much larger radius, leaving the entire $5 \mathrm{~cm}<r<30 \mathrm{~cm}$ region devoid of current $\left(B_{\theta} \propto 1 / r\right)$. The return current in the simulation is at $\approx 8-15 \mathrm{~cm}$, where $B_{\theta}$ deviates from the $1 / r$ behavior and quickly becomes zero. The return magnetic flux in the experiment, on the other hand, is located at $\approx 9-10 \mathrm{~cm}$, very similar to the simulation.

The $B_{\theta}$ due to the axial current in the jet produces a radially outward Lorentz force at the location of the return current. The expansion speed of the return current is determined by the density of the return flux region ( $T$-shell in Figure 4$)$ and the background pressure. The density of the return flux region $n \sim 10^{21} \mathrm{~m}^{-3}$ (Figures 4 and 8 ) is possibly too high compared to the experiment, although the experiment does not have accurate measurements of the low density plasma in the return current region. Also, the background pressure in the experiment $\left(10^{-7}\right.$ torr $\sim 10^{-5}$ Pa for $n \sim 10^{15} \mathrm{~m}^{-3}$ and $T=300 \mathrm{~K}$ ) is much lower than the simulation background pressure $\left(p_{0}=3.2 \mathrm{~Pa}\right.$ for $n=10^{19} \mathrm{~m}^{-3}$ and $T=2 \mathrm{eV}$ ). Numerical investigation has found that the return current extends to a larger radius for a less dense $T$-shell or background. More discussion is given in Sections 5.3 and 6.

Figure 11 plots the 3D global magnetic field structure at $t=29.1 \mu \mathrm{s}$, which shows a typical magnetic tower structure with upward flux along the jet and return flux surrounding the jet. The upward flux is twisted relative to the return flux. Tracing each field line from mid plane, the ratio $B_{\theta} / B_{z}$ is roughly constant along the central jet, and increases rapidly near the jet head because $B_{z}$ becomes zero at the turning point. Combining this figure with Figure 6 panels (E) and (F), we find that at the jet head the poloidal field along the axis can remain comparable to the toroidal field at the jet boundary, although for each field line $B_{\theta} / B_{z}$ always increases. This is because the poloidal field and current do not bend over and return to mid plane at exactly the same height and same radius, i.e., there is no distinct jet head (also see Section 4.1.2). Both $B_{z}$ along the axis and $B_{\theta}$ at the jet boundary decrease gradually in the jet head region, giving a relatively constant ratio between them. The opening angles of the field lines shown in Figure 11 are $5^{\circ}-6^{\circ}$. Calculation shows that a field line starting from $r \sim 0.2$, essentially the boundary of the jet, has an opening angle of $11^{\circ}$; a field line from $r=0.1$ has an opening angle of $4^{\circ}$. It is found in the simulation that the opening angles become smaller as the toroidal field injection continuously accelerates and collimates the jet.

\subsubsection{Alfvén Velocity and Alfvén Surface}

Spruit (2010) categorizes the standard magnetocentrifugal acceleration model (e.g., Blandford \& Payne 1982) into three distinct regions: accretion disk, magnetic dominant region surrounding the central objects, and a distant kinetic dominant region. An Alfvén surface, on which the plasma velocity equals the Alfvén velocity $v_{A} \equiv B / \sqrt{\mu_{0} \rho}$, separates the magnetic dominant region and kinetic dominant region, since the ratio of plasma velocity to Alfvén velocity, $v / v_{A}=\left[\left(\rho v^{2}\right) /\left(B^{2} / \mu_{0}\right)\right]^{1 / 2}$, is the square root of the ratio of kinetic energy to magnetic energy.

Figure 12 plots the distribution of dimensionless Alfvén velocity (top four panels) and $v / v_{A}$ ratio (bottom four panels) in the $r z$ plane at different times. The boundaries of the central jet region and the diffuse pinch region are overlaid on the lower right panel. The figure shows that $v_{A}$ is always high in the diffuse pinch region due to the low density and strong toroidal field. In the central jet, $v_{A}$ remains roughly constant because of the quasi-constant density and magnetic field configuration. The high Alfvén velocity region increases in volume together with the jet propagation.

The $v / v_{A}$ distribution plots show that the Alfvén surface, denoted by the innermost $v=v_{A}$ contour curve, is also expanding. In the $+z$ direction, the Alfvén surface propagates from $0.5 R_{0}=9 \mathrm{~cm}$ at $t=11.6 \mu \mathrm{s}$ to $1.5 R_{0}=27 \mathrm{~cm}$ at $t=29.1 \mu \mathrm{s}$ at a speed of $\approx 10 \mathrm{~km} \mathrm{~s}^{-1}$, similar to the jet propagation speed. Along the central axis, the $v / v_{A}$ ratio gradually increases from $\ll 1$ at jet base to $\sim 1$ at jet head, 
Poloidal flux contours, Argon shot\#12780, z=15 cm

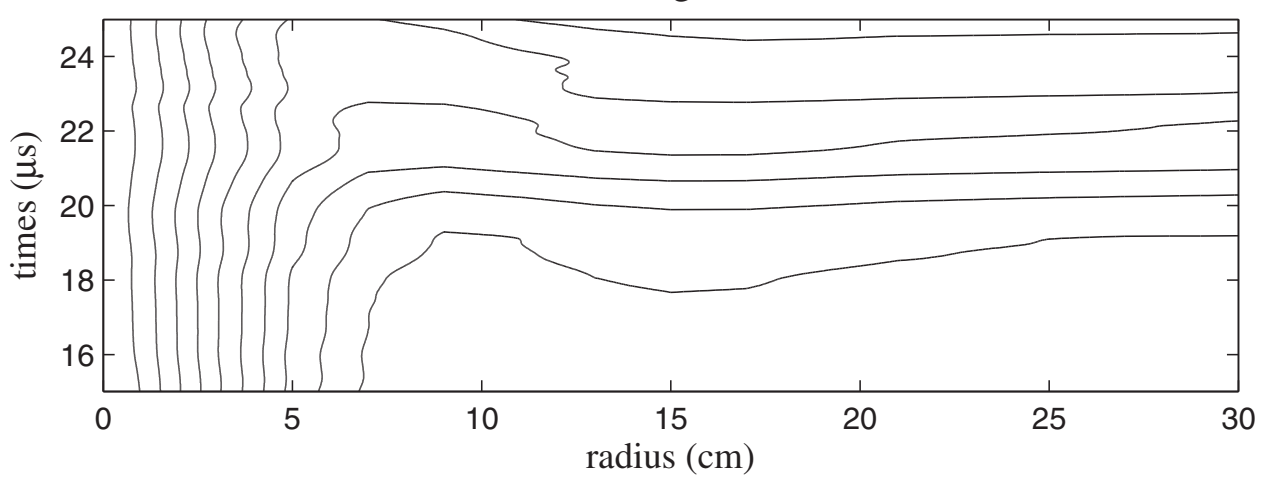

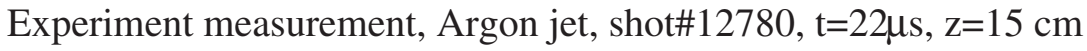

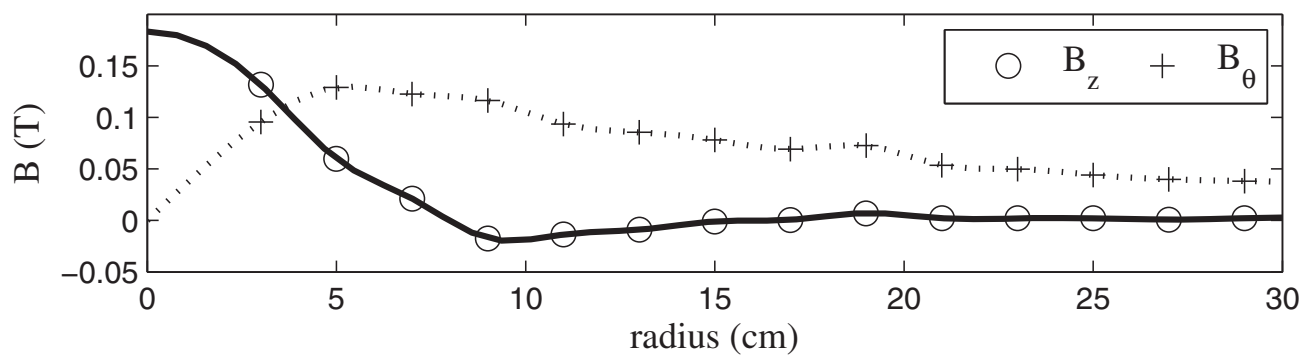

Simulation, Argon jet, $\mathrm{t}=24 \mu \mathrm{s}, \mathrm{z}=14.4 \mathrm{~cm}+\mathrm{z}_{\text {foot }}$

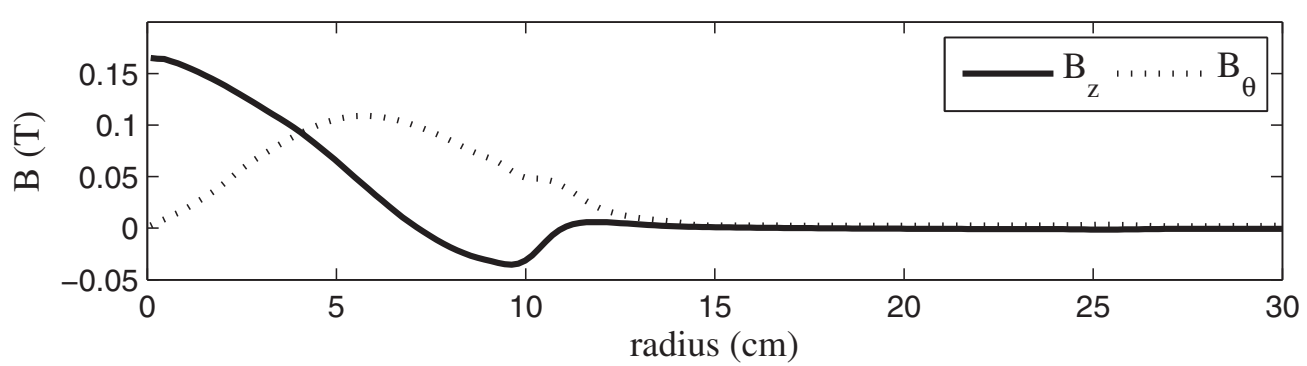

Figure 10. Top panel: poloidal magnetic field contours inferred from the MPA measurements from $t=15 \mu$ s to $t=25 \mu$ s. Mid and bottom panels: magnetic field in axial (heavy solid curves) and azimuthal (dotted curves) directions measured in the experiment (mid panel) and in the simulation (bottom panel). The experimental measurements (top two panels) are obtained in argon jet experiment shot 12780. This experiment jet remains quasi-axisymmetric at $t=22 \mu \mathrm{s}$.

and becomes $\gg 1$ at the hydro shock which has no magnetic field. According to Figures 4, 6, and 9, the magnetic tower, wherein dense plasma encloses strong axial magnetic field $B_{z}$ and axial current $J_{z}$, is inside the Alfvén surface. We point out here that the entire jet collimation and propagation dynamics is an integrated process. It is inappropriate to characterize the jet as a hydrodynamic jet or magnetized jet simply based on the local $v / v_{A}$ ratio, because the Alfvén surface is also expanding. Although the kinetic energy of the global system extends beyond the Alfvén surface in Figure 12, the magnetic tower is still an MHD-driven jet. Outside the Alfvén surface, according to Figure 6, both the poloidal and toroidal components of the magnetic field decrease rapidly. The entire diffuse pinch region always has a relatively low $v / v_{A}$ ratio. Outside the Alfvén surface, there is another $v_{A}=v$ contour expanding outward, which indicates the hydrodynamic shock. This is essentially the boundary of the entire large-scale jet structure. Outside this structure, both $v$ and $v_{A}$ are zero.

\subsection{Bernoulli Equation in MHD Driven Flow}

We have shown in detail the process of jet collimation and propagation resulting from the MHD mechanism. In
Section 4.1.2, we have demonstrated that the jet gains its kinetic energy from magnetic energy; kinetic energy dominates near the jet head while magnetic energy dominates near the jet base. This has been quantitatively verified in the experiment.

Assuming that the Lorentz force balances the thermal pressure gradient in the radial direction, an axisymmetric model was proposed by Kumar \& Bellan (2009) and Kumar (2009) to study the non-equilibrium steady-state flow along the axial direction. The model claims that a Bernoulli-like quantity involving the toroidal magnetic energy remains constant along the jet, i.e.,

$$
\frac{\partial}{\partial z}\left[\rho v_{z}^{2}+\frac{B_{\theta, a}^{2}}{\mu_{0}}\left(1-\frac{r^{2}}{2 a^{2}}\right)\right]=0,
$$

where $a$ is the jet radius and $B_{\theta, a}=\mu_{0} I /(2 \pi a)$ is the toroidal field strength at the jet boundary. Evaluating the expression at $r=0$ gives

$$
\rho v_{z}^{2}+\frac{B_{\theta, a}^{2}}{\mu_{0}}=\rho v_{z}^{2}+\frac{\mu_{0} I^{2}}{4 \pi a^{2}}=\text { const }
$$

which is a Bernoulli-like equation. At $z \sim 0$, the axial velocity $v_{z} \approx 0$ so the magnetic energy dominates. At the jet head, 

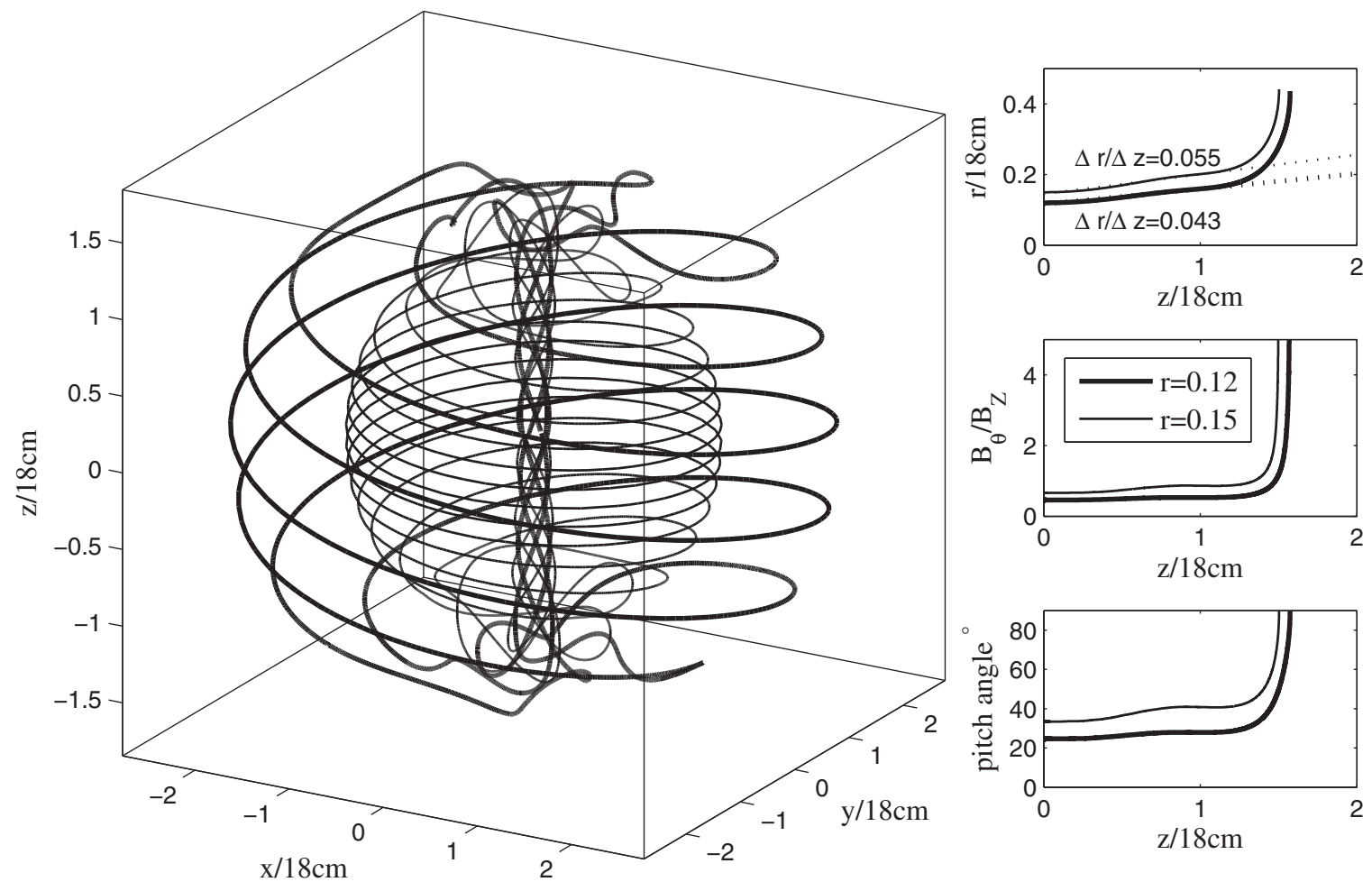

Figure 11. Left: 3D magnetic field structure of the simulation jet. The structure is composed by two groups of field lines starting from mid plane at $r=0.12(2.16 \mathrm{~cm})$ and $r=0.15(2.7 \mathrm{~cm})$. Each group contains four field lines azimuthally equally spaced starting at same radius. Upper right: radial location of each fieldline at a different height. Linear regression within $0 \leqslant z \leqslant 1.2$ gives $\Delta r / \Delta z=0.043$ with $R^{2}=0.981$ for field lines starting from $r=0.12$, and $\Delta r / \Delta z=0.055$ with $R^{2}=0.980$ for field lines starting from $r=0.15$. These correspond to opening angles $\theta=2 \arctan (\Delta r / \Delta z)=4.9^{\circ}$ and $6.3^{\circ}$ for the two groups of field lines, respectively. Mid right: $B_{\theta} / B_{z}$ along the field line from mid plane to jet head. Lower right: the pitch of the magnetic field $\theta \equiv \arctan \left(B_{\theta} / B_{z}\right)$ in degree. In all three subplots, the thick curves represent fieldlines starting from $(r=0.12, z=0)$ and the thin curves represent fieldlines from $(r=0.18, z=0)$. The fieldlines are obtained at $t=0.5$ or $29.1 \mu \mathrm{s}$.
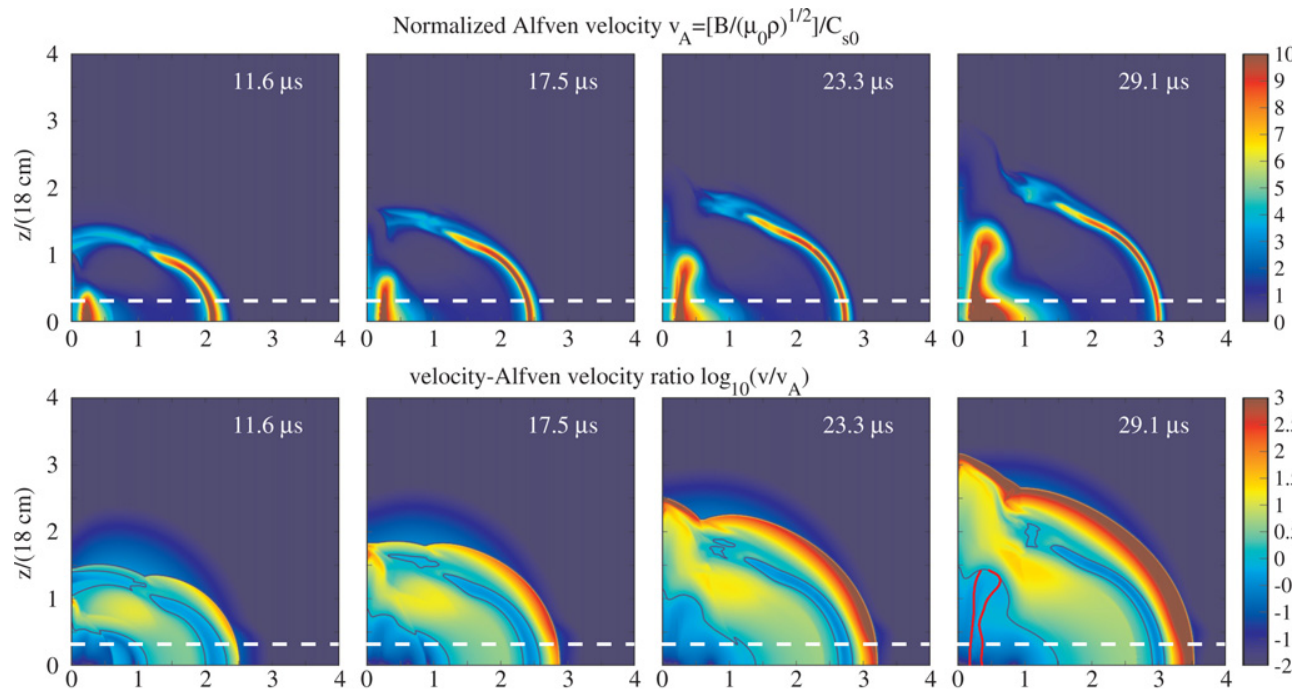

velocity-Alfven velocity ratio $\log _{10}\left(\mathrm{v} / \mathrm{v}_{\mathrm{A}}\right)$
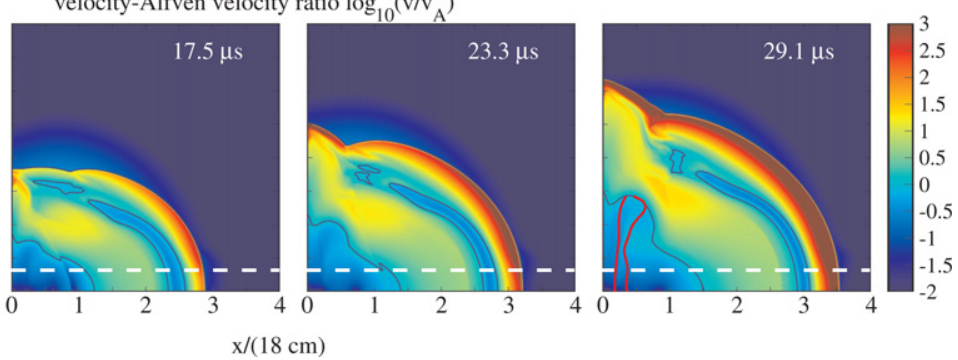

Figure 12. Alfvén velocity $v_{A}$ and velocity to Alfvén velocity ratio $v / v_{A}$ in $r z$ plane at different times. Top four panels: the color map of dimensionless Alfvén velocity $v_{A} / C_{s 0}=\left[B / \sqrt{\mu_{0} \rho}\right] / C_{s 0}\left(C_{s 0}\right.$ given in Table 1). Bottom four panels: the color map of $\log _{10}\left(v / v_{A}\right)$ with $v=v_{A}$ contours (blue curves). The lower right panel is overlaid by two red curves. The one at smaller radius from $z=0$ to $z=1.6$ represents the contour of maximum $B_{\theta}$ at each height, and is the boundary between the central jet region and diffuse pinch region. The red curve at larger radius is the $J_{z}=0$ contour, that separates the diffuse pinch region and the return flux region.

(A color version of this figure is available in the online journal.)

$B_{\theta, a} \approx 0$ so the kinetic energy dominates. This is consistent with the analysis in Section 4.1.2. Evaluating Equation (29) at $z=0$ and at the jet head yields

$$
\left.\left.v_{z}\right|_{\text {jet head }} \simeq \frac{I}{2 \pi a} \sqrt{\frac{\mu_{0}}{\rho}}\right|_{z=0} \propto \frac{I}{\sqrt{\rho}} .
$$

Kumar \& Bellan (2009) and Kumar (2009) report quantitative experimental measurements and show that the axial velocity of the MHD-driven plasma jet is linearly proportional to the poloidal current, and inversely proportional to the square root of the jet density. Therefore, Equation (30), a direct corollary of Equation (28), has been verified by the experiment. 

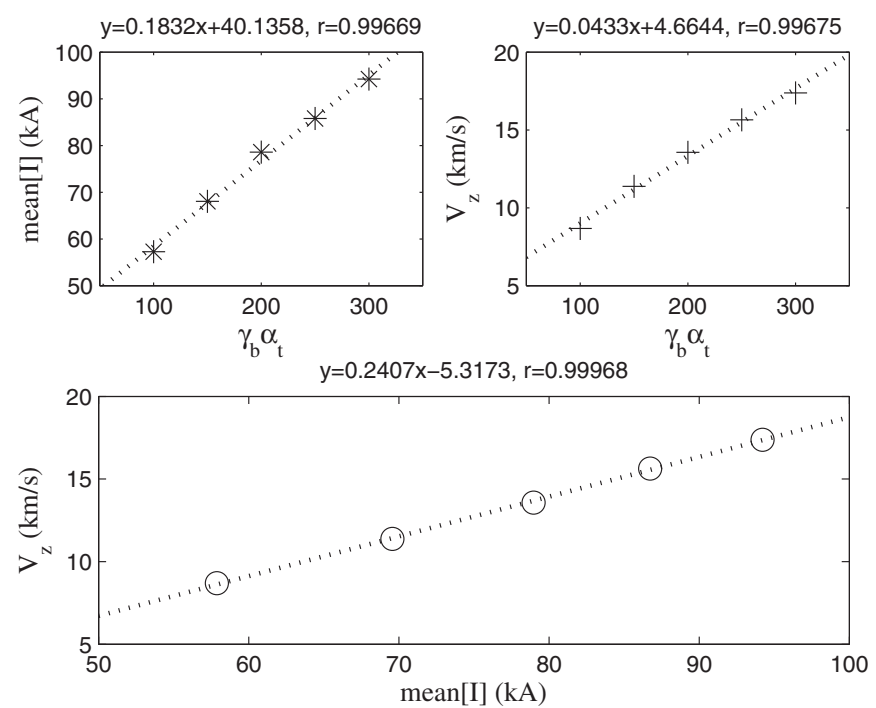

Figure 13. Jet velocity dependence in the simulation. Upper left: time-averaged total poloidal current for different injection rate $\gamma_{b} \alpha_{t}$ (asterisk symbols). Upper right: the averaged jet velocity at different injection rate $\gamma_{b} \alpha_{t}$ (plus symbols). Bottom: jet velocity vs. total poloidal current (open circle symbols). The averaging period is the time the jet head spends traveling from $z=30 \mathrm{~cm}$ to $60 \mathrm{~cm}$. For each subplot, linear regression is performed (dotted lines) and the results are presented as the title.

Equation (30) can be understood from a semi-quantitative analysis. Since the injected Poynting flux or toroidal magnetic field energy will ultimately be used to accelerate the jet, an energy equal-partition gives $B_{\theta}^{2} \sim \rho v_{z}^{2}$. Hence $v_{z} \sim B_{\theta} / \sqrt{\rho} \sim$ $I / \sqrt{\rho}$. Similar analysis and scaling can also be found in Lynden-Bell (1996, 2003), Uzdensky \& MacFadyen (2006), and Hennebelle \& Fromang (2008).

We now use the simulation to investigate this relation.

\subsubsection{Jet Velocity Dependence on the Poloidal Current}

We use the same initial conditions as in Section 4.1, and the same localized toroidal field injection with the localization factor $A=9$. However, in order to control the total poloidal current, we use constant injection rates $\gamma_{b} \alpha_{t}$ throughout the simulation. Five simulations are performed with different timeindependent injection rates over a wide range: $\gamma_{b} \alpha_{t}=100,150$, 200,250 , and 300 . The average jet velocity is computed using the time the jet head takes to travel from $z=30 \mathrm{~cm}$ to $60 \mathrm{~cm}$ $(z=1.67$ to 3.33 in reduced units). Here, we define the location of the jet head as being where the plasma density drops below $10^{21} \mathrm{~m}^{-3}$ along the $z$ axis. According to Figures 4 and 6 , this definition gives a sufficiently consistent estimation of the jet head location. The total poloidal current is also averaged over the same period. Figure 13 shows that both the jet velocity and the time-averaged total poloidal current are proportional to the toroidal field injection rate $\gamma_{b} \alpha_{t}$. Thus, the jet velocity is indeed proportional to the poloidal current.

\subsubsection{Jet Velocity Dependence on the Jet Density}

Kumar \& Bellan (2009) and Kumar (2009) find that under the same experimental configuration, a deuterium plasma jet always propagates at a speed $=0.73 \approx 1 / \sqrt{2}$ times the speed of a hydrogen plasma jet. Hence $v_{z} \propto 1 / \sqrt{\mu} \sim 1 / \sqrt{\rho}$ is verified. In the simulation, this dependence is already incorporated by the normalization process in Section 3.1. Note that the simulation time unit is defined as

$$
t_{0} \equiv \frac{R_{0}}{C_{s 0}} \propto \frac{1}{C_{s 0}}
$$

and

$$
C_{s 0}^{2} \propto \frac{1}{m_{i}} \propto \frac{1}{\mu}, \quad \mu \equiv \frac{m_{i}}{m_{H}},
$$

so the simulation time unit is proportional to $\sqrt{\mu}$. Therefore, the simulation velocity unit is proportional to $1 / \sqrt{\rho}$.

Given that $n \approx 10^{22} \mathrm{~m}^{-3}$ and $a \approx 4 \mathrm{~cm}$, Equation (30) predicts $v_{z} / I \simeq \sqrt{\mu_{0} / \rho_{0}} /(2 \pi a)=0.244 \mathrm{~m} /(\mathrm{s} \cdot \mathrm{A})=$ $0.244 \mathrm{~km} \mathrm{~s}^{-1} / \mathrm{kA}$, which is consistent with the linear regression results given in the bottom panel of Figure 13.

\subsubsection{A Direct Illustration of MHD Bernoulli equation}

In fact, Equation (28) can be easily verified directly by the simulation. Evaluating the equation at the jet radius $r=a$ gives

$$
\frac{\partial}{\partial z}\left(\rho v_{z}^{2}+\frac{B_{\theta, a}^{2}}{2 \mu_{0}}\right)=\left.0 \Rightarrow\left(e_{k}+e_{B_{\mathrm{tor}}} / 2\right)\right|_{\text {jet radius }}=\text { const }
$$

where the kinetic energy density is $e_{k} \equiv \rho v_{z}^{2} / 2$ and the toroidal magnetic field energy density is $e_{B_{\text {tor }}} \equiv B_{\theta}^{2} / 2 \mu_{0}$.

We choose the $\gamma_{b} \alpha_{t}=200$ simulation presented in Section 4.2.1 and plot the 1D profile of $\left(e_{k}+e_{B_{\text {tor }}} / 2\right)$ along the jet radius and the cross-sectional two-dimensional view of $\left(e_{k}+e_{B_{\text {tor }}} / 2\right)$ and density/flux in Figure 14 . The three plots directly illustrate that at any given time after jet collimation is completed, $\left(e_{k}+e_{B_{\text {tor }}} / 2\right)$ is constant on the boundary of a magnetic tower jet through the entire jet body.

Having cross-checked the jet velocity dependence on poloidal current and density using experiments, simulation, and analytical theory, and also demonstrated that Equation (33) holds along the jet in the simulation, we conclude that Equation (30), and more generally, the MHD Bernoulli Equation (28) are true for magnetic tower jets, such as the Caltech experimental plasma jet and possibly actual astrophysical jets.

\section{SENSITIVITY TO IMPOSED SIMULATION CONDITIONS}

The numerical simulations presented in Section 4 are based on a number of imposed conditions, including initial mass distribution, background pressure, initial poloidal field, toroidal field injection rate and toroidal field injection volume (factor $A$ ). As discussed in Sections 3 and 4, the initial poloidal field flux and toroidal field injection rate are selected strictly on the experiment properties. The initial mass distribution in simulation is similar to the real experiment case. We now examine how our key conclusions depend on these imposed conditions.

We perform another eight simulations with exactly the same conditions as the simulation presented in Section 4.1 (referred as the "original" simulation or simulation $\mathrm{A}$ in the following discussion), except for one different condition. The density distribution and poloidal field configuration at $t=29.1 \mu \mathrm{s}$ of these eight simulations are plotted in Figure 15 together with the original simulation.

\subsection{Background Condition}

The original simulation has a background plasma particle number density $n_{\text {background }}=1$, or $10^{19} \mathrm{~m}^{-3}$, about 

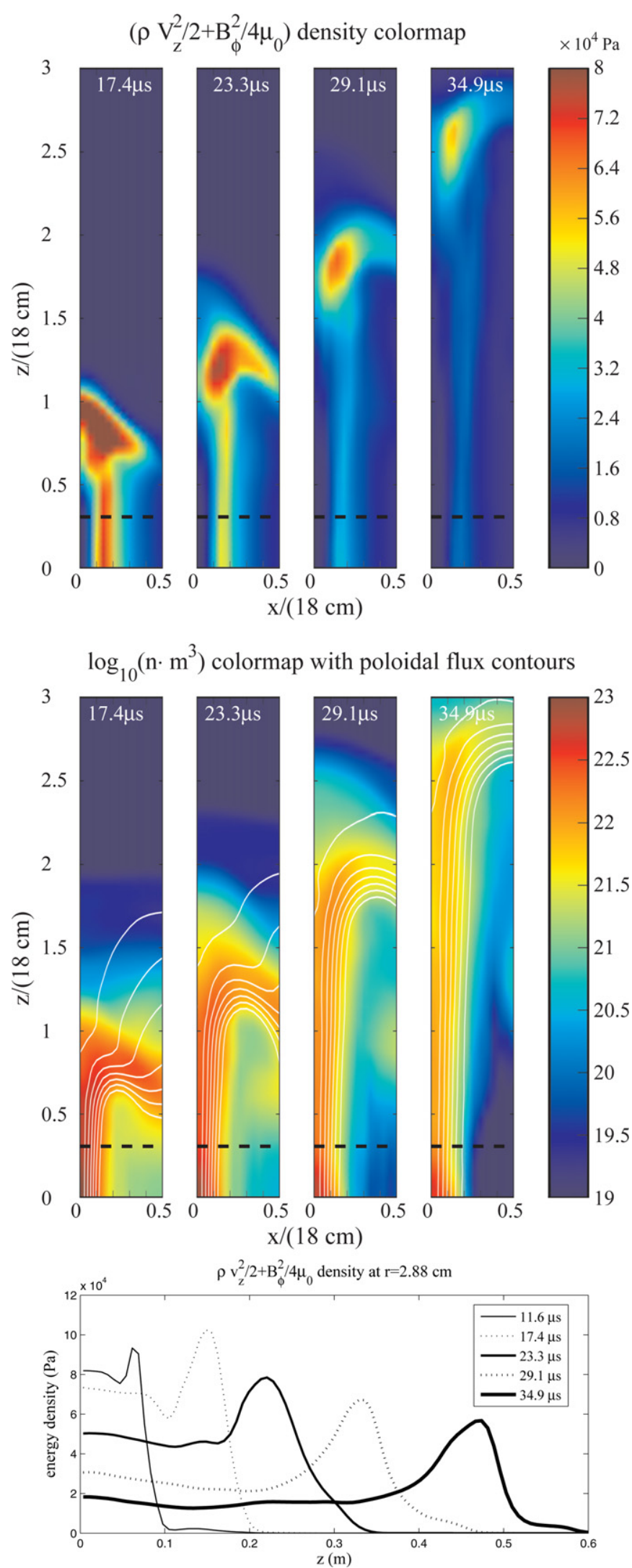

Figure 14. Top panel: cross-sectional view of $\left(e_{k}+e_{B_{\text {tor }}} / 2\right)$ energy density on the $x z$ plane $(y=0)$ from $17.4 \mu$ s to $34.9 \mu \mathrm{s}$. Mid panel: cross-sectional view of density distribution (log scale) at the same times as used for the plots in the top panel. Azimuthally averaged poloidal flux contours are overlaid. Note that the jet radius to length ratio has dropped to $\sim 1: 20$ at $t=34.9 \mu$ s. Bottom panel: $\left(e_{k}+e_{B_{\mathrm{tor}}} / 2\right)$ at $r=2.88 \mathrm{~cm}$ along the $z$ direction at different times. The plots are generated from the $\gamma_{b} \alpha_{t}=200$ simulation in Section 4.2.1.

(A color version of this figure is available in the online journal.)
$10^{3}-10^{4}$ times less dense than the central jet (panel (A) in Figure 15). In the experiment, this number is $10^{7}-10^{8}$. However, as long as the background density is significantly lower than the plasma of interest, the dynamics of the central jet should not be affected.

This is verified by simulations $\mathrm{B}$ and $\mathrm{C}$, which have $n_{\text {background }}=0.1$ and 10 , respectively. Comparing A, B, and $\mathrm{C}$, they show no difference in the central jet and the vicinity. The hydro shock and return flux at very large radii, however, are indeed affected by the different background conditions. Consistent with the discussion in Section 3.2.2, Sections 4.1.2 and 4.1.3, a lower background pressure imposes a weaker restriction to the expansion of the system.

In an astrophysics situation, the density difference between the central jet and ambient environment (ISM/intergalactic medium) is expected to be less than in the experiment and the shock structure and the return flux are expected to be somewhat different. With a significant background pressure, the expansion of return flux and current can be highly constrained. If the return flux and current are sufficiently near the center jet, they can influence the jet stability properties. This is similar to how a conducting wall surrounding a current-carrying plasma tube can prevent the plasma against from developing a kink instability (e.g., Bellan 2006).

\subsection{Toroidal Field Injection Condition}

The toroidal field injection condition is subjected to two major possible variations: injection rate and injection volume.

The injection rate affects the total poloidal current and therefore affects the jet velocity according to Equation (30). In Section 4.2, we have addressed this issue by performing five simulations with different injection rates. Figure 13 shows that jet velocity is proportional to the toroidal injection rate.

Injection volume is determined by the injection factor $A$ (Section 3.3.1). We already pointed out that the factor $A$ does not alter the total poloidal current associated with the toroidal field. Simulations D and E shown in Figure 15 are performed with $A=3$ and $A=6$, respectively. At $z=1$, the factor $e^{-A z^{2}}=0.05,2.5 \times 10^{-3}$ and $1.2 \times 10^{-4}$ for $A=3$ (D), 6 (E) and 9 (A), respectively. Even with such enormous differences, the plasmas in simulations A, D, and E evolve in very similar ways. This is because the injected toroidal field is able to emerge into the propagating jet rapidly, no matter where the field is initially injected (see also in Figures 6, 7 and 9).

A notable difference for different $A$ factors is the behavior of the hydro shock and remote return flux. This is because toroidal injection with a smaller $A$ gives larger direct field injection at larger distance and low density region, and therefore gives rise to a faster expanding shock and return flux.

The $A$ factor determines the thickness of the effective engine region. In the experiment and astrophysics cases, the engine region is expected to be limited to the electrodes or the vicinity of central objects. Ideally, a toroidal injection with a larger $A$ factor provides better approximation to the real cases. However, the $A$ factor has little effect on the dynamics of the central jet.

\subsection{Initial Mass Distribution}

As shown in Sections 3 and 4, the jet is created as a result of a gradient along the $z$ direction of the pressure associated with the toroidal magnetic field. Therefore, the initial mass distribution should not be crucial in the jet dynamics. 
$\log _{10}\left(\rho / \mathrm{m}^{-3}\right)$ color map with poloidal flux contours at $\mathrm{t}=29.1 \mu \mathrm{s}$, different simulation conditions
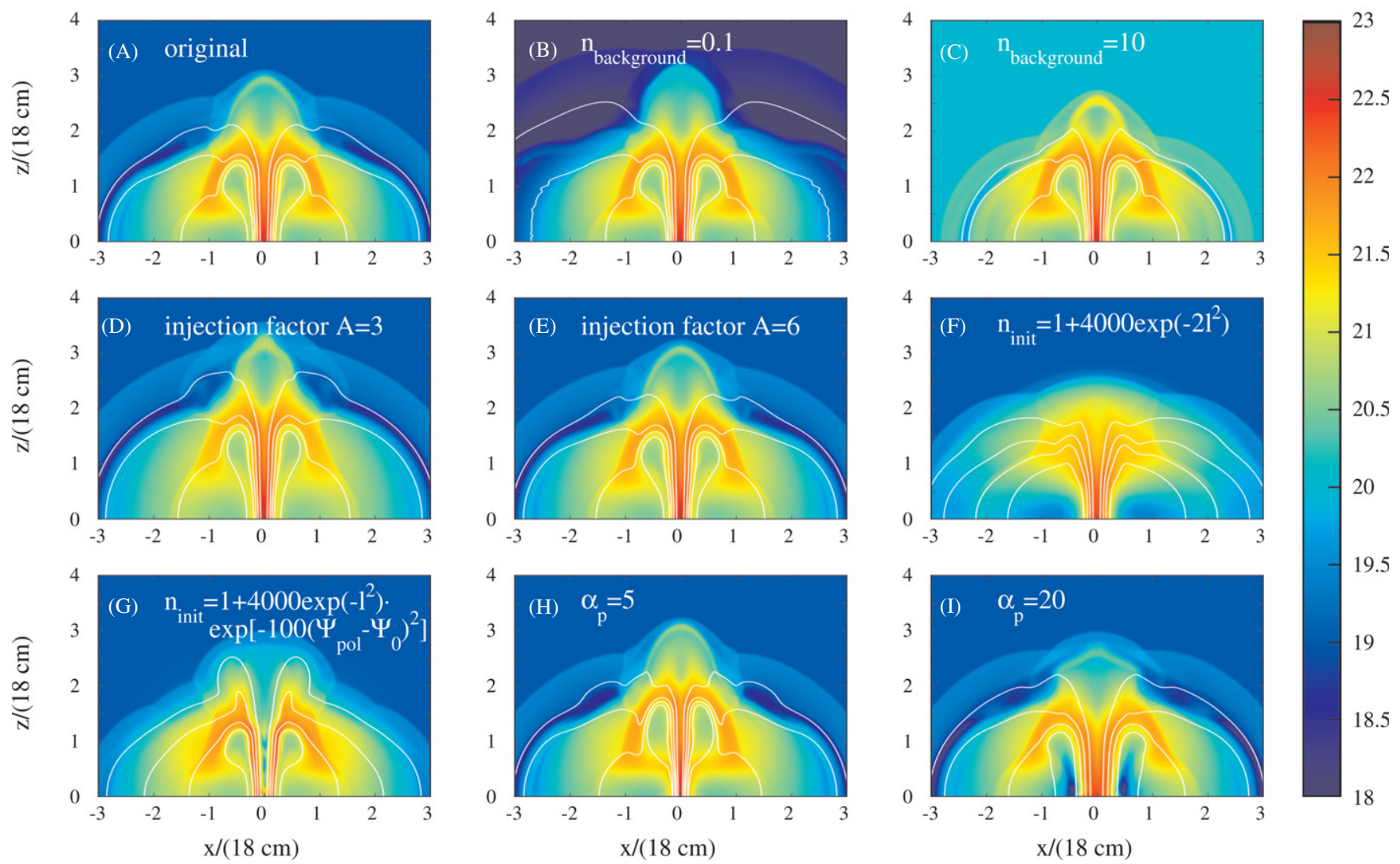

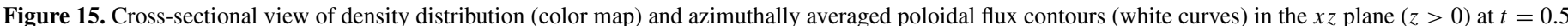

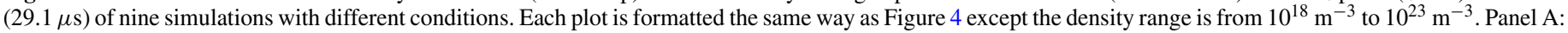

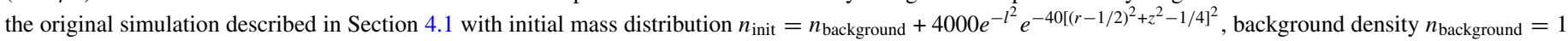

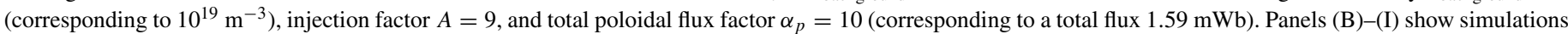

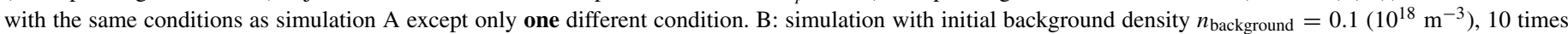

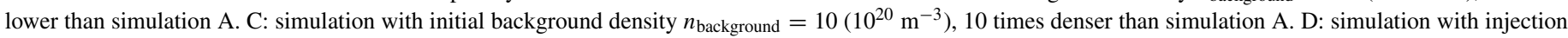

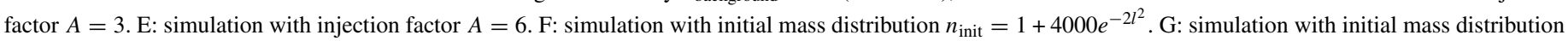

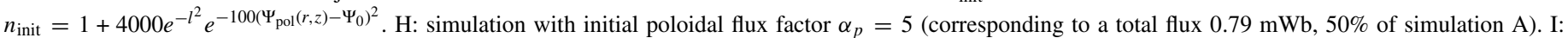

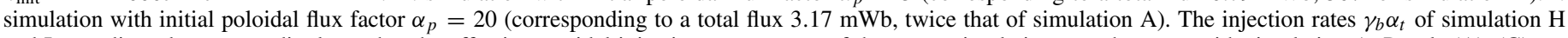

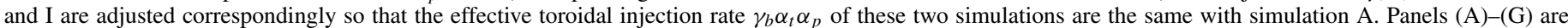

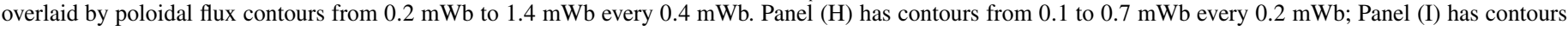
from $0.4 \mathrm{mWb}$ to $2.8 \mathrm{mWb}$ every $0.8 \mathrm{mWb}$.

(A color version of this figure is available in the online journal.)

Simulation $\mathrm{F}$ adopts a very different initial mass distribution $n_{\text {init }}=1+4000 e^{-2 l^{2}}$, where $l^{2}=r^{2}+z^{2}$. A central jet is created with a similar radius and slower speed. Further investigation shows that the well-collimated portion extends from $z \approx 0.8$ to 1.1 in the next $6 \mu \mathrm{s}$. The return flux manages to expand further because of the relative low density at large radii initially. The general jet behaviors are consistent with simulation A.

Simulation $\mathrm{G}$ takes an initial mass distribution very similar to the real experiment case, $n_{\text {init }}=1+4000 e^{-l^{2}} e^{-\delta\left(\Psi_{\mathrm{pol}}(r, z)-\Psi_{0}\right)^{2}}$ with $\delta=100$ (see Section 3.2.2). The central region is initially filled with low density plasma. In the experiment, fast magnetic reconnection allows the magnetic field to diffuse into the center along with the plasma. However, in ideal MHD theory, reconnection is forbidden. As shown in panel (G) of Figure 15, a hollow jet is eventually formed. The axis magnetic field is stronger along the axis than simulation $\mathrm{A}$, because there is no dense plasma in the center helping the poloidal flux against compression of the toroidal pinch. Because the plasma is initially distributed parallel to the poloidal field, simulation $\mathrm{G}$ shows a better alignment between plasma and poloidal flux compared to simulation A.

Although the detailed form of initial mass distribution does not significantly affect the formation of the central magnetic tower jet, it can at later times impact the density distribution at larger radius, such as return flux region, and therefore can potentially influence the expansion of the return current. Three additional simulations A2, A3, and A4 are performed which are the same as simulation A (original one) except that there is less dense plasma at either larger radius or larger height. Table 2 lists the detailed function of initial mass distribution and the location of return current at $z=20 \mathrm{~cm}$ for each simulation. Max $B_{\theta}$ in Table 2 is the toroidal field strength at the central jet surface. The return flux region ( $T$-shell) of A2-A4 is less dense than that of simulation A. This is because initially there was less dense 
Table 2

Location of the Return Current of Simulations with Different Initial Density Distributions

\begin{tabular}{llccc}
\hline \hline Simulation & Initial Mass Distribution & $\begin{array}{c}\operatorname{Max} B_{\theta} \\
(\mathrm{T})\end{array}$ & $\begin{array}{c}R_{J_{z}=0} \\
(\mathrm{~cm})\end{array}$ & $\begin{array}{c}\bar{R}_{J_{z}<0} \\
(\mathrm{~cm})\end{array}$ \\
\hline $\mathrm{A}$ & $1+4000 f(r, z) e^{-r^{2}-z^{2}}$ & 0.119 & 7.3 & 11.7 \\
$\mathrm{~B}$ & $0.1+4000 f(r, z) e^{-r^{2}-z^{2}}$ & 0.107 & 8.3 & 11.9 \\
$\mathrm{~F}$ & $1+4000 e^{-2 r^{2}-2 z^{2}}$ & 0.083 & 9.8 & 22.6 \\
$\mathrm{~A} 2$ & $1+4000 f(r, z) e^{-r^{2}-4 z^{2}}$ & 0.123 & 9.2 & 16.5 \\
$\mathrm{~A} 3$ & $1+4000 f(r, z) e^{-2 r^{2}-z^{2}}$ & 0.091 & 9.0 & 14.5 \\
A4 & $1+4000 f(r, z) e^{-2 r^{2}-4 z^{2}}$ & 0.100 & 10.5 & 21.6 \\
\hline
\end{tabular}

Notes. Initial density distribution at $z=20 \mathrm{~cm}$ and $t=24.4 \mu \mathrm{s}$ for Simulations A, B, A2-A4, and $t=27.9 \mu$ for Simulation F. Function $f(r, z)=e^{-40\left[(r-1 / 2)^{2}+z^{2}-1 / 4\right]^{2}} . R_{J_{z}=0}$ is the radius where axial current changes sign and $\bar{R}_{J_{z}<0}$ is the averaged location of the return current, defined as $\bar{R}_{J_{z}<0} \equiv\left(\sum r\left|J_{z}\right|^{2}\right) /\left(\sum\left|J_{z}\right|^{2}\right)$ for all negative $J_{z}$. The numbers for simulation $\mathrm{F}$ are obtained at $t=27.9 \mu \mathrm{s}$ when the jet has a similar height as the other simulation jet at $t=24.4 \mu$ s (see Figure 15 panel (A) and (F)).

plasma at larger radius or height. As expected, the return current of A2-A4 expands faster than does simulation A. With a lower background pressure, simulation $\mathrm{B}$ also has a faster expanding return current than $\mathrm{A}$ does.

It is found that all of these simulations produce similar magnetic/kinetic profiles in the central region, although their return current profiles differ significantly. This is because, according to Ampere's Law, there is no magnetic field generated by the return current at the central jet location. In both the simulation and experiment, there is no boundary condition constraining the radius of zero net current and hence the return current radius can expand from the MHD force. The return flux region of simulation A expands at speed $v_{r} \approx v_{z} \approx 6 \mathrm{~km} \mathrm{~s}^{-1}$ at $t=29.1 \mu \mathrm{s}$ (Figure 8). This is comparable with the Alfvén velocity $V_{A} \sim 15 \mathrm{~km} \mathrm{~s}^{-1}$ in the diffuse pinch region between the central jet and the return flux/current.

\subsection{Initial Poloidal Flux}

Compression of the poloidal flux tends to oppose the pinching force of the toroidal field. Simulations $\mathrm{H}$ and I verify this with $50 \%$ and $200 \%$ initial poloidal flux compared to simulation A. Panels $(\mathrm{H})$ and (I) of Figure 15 show that with less poloidal flux, the jet has a smaller radius and propagates faster; with doubled poloidal flux, on the other hand, the plasma struggles to compress the poloidal field, resulting in a much wider and slower jet.

In non-axisymmetric situations, the poloidal flux is expected to impact the stability properties of the current-conducting jet. Experiment investigation involving changing the ratio between poloidal current and poloidal flux, known as "gun parameter," shows that the jet undergoes MHD kink instability when the classical Kruskal-Shafranov threshold is satisfied (Hsu \& Bellan 2003, 2005).

In summary, we have shown here how different conditions affect the simulation results. The conditions that directly determine the jet dynamics, such as initial poloidal flux and toroidal injection rate, are selected strictly from the actual experiment conditions. Those conditions that only affect the dynamics of return flux and the hydro shock, such as background pressure, initial mass distribution, and toroidal injection volume, can be subject to relatively large variations without significantly influencing the jet dynamics.

\section{SUMMARY AND DISCUSSION}

We have presented MHD numerical simulations of the Caltech plasma jet experiment using a magnetic tower model similar to Li et al. (2006). By having a purely toroidal magnetic injection localized around the $z=0$ plane, the simulation jet gains energy and helicity in a manner analogous to the electrode-driven experimental jet, or to astrophysical jets driven by accretion disks. In the simulation, the injected toroidal field near $z=0$ is efficiently carried through the jet and is responsible for generating the pinch force that collimates both the plasma and the embedded poloidal magnetic field. The gradient of the collimation force along the jet boundary, or equivalently, the gradient of toroidal magnetic field energy in the $z$ direction, is responsible for accelerating the jet. Magnetic to kinetic energy conversion is verified in the simulation along with the experiment.

The simulation jet agrees quantitatively with the experimental jet in numerous ways, including the energy partition/evolution, current/voltage, jet radius, axial profile, magnetic field structure, and jet velocity scaling. Furthermore, by using the unit systems given in Table 1, the simulation results can easily be made dimensionless and then converted to astrophysical scales.

One of the most significant outcomes of this simulation work is the validation of using terrestrial laboratory experiments to study astrophysical jets. Although it is not feasible to experimentally reproduce every single aspect of an astrophysical jet, by careful experiment design it is possible to replicate many of the most important mechanisms that govern the jet dynamics. Also, the experimental investigation shares common advantages with the numerical simulation such as reproducibility, freedom in parameter space and possibility of in-situ measurement. This paper suggests that combining observation, theoretical modeling and laboratory experiments helps understand the nature of magnetically driven plasma flows.

We emphasize here that the simulation does not prove that the experimental jets are exactly the same as astrophysical jets. Neither the simulation nor the experiment is expected to reproduce every detail of a theoretical model or an astrophysical jet. However, the fact that an astrophysical magnetic tower model can be used to simulate laboratory experiments suggests that the experiment shares several important similarities with astrophysical jets, such as the collimation and propagation mechanisms. Furthermore, consideration of any discrepancies between experiment and simulation help understand the underlying physics.

In both the experiment and simulation, there is no boundary condition or other restriction on the expansion of the return current/flux. The return current/flux expands at a velocity comparable to Alfvénic velocity but the dynamics of the central magnetic tower jet is not influenced by the return current/ flux. In astrophysical situations where the background pressure is important (e.g., Lynden-Bell 1996; Li et al. 2001), free expansion of the return current/flux can be inhibited, resulting in a small or null diffuse pinch region, i.e., the return current/flux could be snugly on the surface of the central jet (e.g., Sherwin \& Lynden-Bell 2007; Nakamura et al. 2007). In this situation, most of the toroidal field energy is inside the central jet so the jet is expected to be more efficiently collimated and accelerated for a fixed amount of toroidal energy. Meanwhile, an extremely dense return flux region closed to the jet could act like a wall that would stabilize the central jet.

The simulation presented in this paper mainly addresses jet launching and acceleration mechanisms, i.e., jet collimation, propagation and energy conversion, and considers only 
axisymmetric dynamics. No asymmetric perturbation is introduced initially or during the simulation. The simulation jets, theoretically vulnerable to kink instability, remain quasiaxisymmetric and stable. However, preliminary investigation has been able to produce kink instability in the simulation, by using a perturbed initial mass distribution. In the experiment, due to the inevitable imperfectly symmetric laboratory condition, the jet always undergoes kink instability when the classic Kruskal-Shafranov condition is satisfied (Hsu \& Bellan 2003, 2005). In some cases, when the kinked plasma grows exponentially fast and accelerates away from the central axis, a lateral Rayleigh-Taylor instability is induced on the inner boundary of the jet. The Rayleigh-Taylor instability further induces a fast magnetic reconnection that breaks the jet in the middle, and removes some magnetized jet segment from the electrodeattached jet segment (Moser \& Bellan 2012a). Astrophysical jets in a similar situation, e.g., kink instability or other lateral acceleration, might also be susceptible to this secondary instability. Numerical investigation of this Rayleigh-Taylor instability is underway.

The experimental program at Caltech is supported by the NSF/DOE Partnership in Plasma Science. H.L. is grateful to Stirling Colgate, Ken Fowler, and Ellen Zweibel for discussions. H.L. and S.L. are supported by the LANL/LDRD and Institutional Computing Programs at LANL and by DOE/Office of Fusion Energy Science through CMSO.

\section{REFERENCES}

Bellan, P. M. 2000, Spheromaks: A Practical Application of Magnetohydrodynamic Dynamos and Plasma Self-Organization (London: Imperial College Press)

Bellan, P. M. 2003, PhPl, 10, 1999

Bellan, P. M. 2006, Fundamentals of Plasma Physics (Cambridge: Cambridge Univ. Press)

Bellan, P. M., Livio, M., Kato, Y., et al. 2009, PhP1, 16, 041005

Berger, M. A. 1999, PPCF, 41, B167

Blandford, R. D., \& Payne, D. G. 1982, MNRAS, 199, 883

Ciardi, A., Lebedev, S. V., Frank, A., et al. 2007, PhP1, 14, 056501
Ciardi, A., Lebedev, S. V., Frank, A., et al. 2009, ApJL, 691, L147

Clausen-Brown, E., Lyutikov, M., \& Kharb, P. 2011, MNRAS, 415, 2081

Colgate, S. A., Fowler, T. K., Li, H., \& Pino, J. 2014, ApJ, 789, 144

Ferrari, A. 1998, ARA\&A, 36, 539

Finn, J., \& Antonsen, T. 1985, CoPPC, 9, 111

Hardee, P. 2008, JPhCS, 131, 012052

Hartigan, P., \& Hillenbrand, L. 2009, ApJ, 705, 1388

Hennebelle, P., \& Fromang, S. 2008, A\&A, 477, 9

Hsu, S. C., \& Bellan, P. M. 2002, MNRAS, 334, 257

Hsu, S. C., \& Bellan, P. M. 2003, PhRvL, 90, 215002

Hsu, S. C., \& Bellan, P. M. 2005, PhPl, 12, 032103

Junor, W., Biretta, J. A., \& Livio, M. 1999, Natur, 401, 891

Kulsrud, R. M. 2005, Plasma Physics for Astrophysics (Princeton, NJ: Princeton Univ. Press)

Kumar, D. 2009, PhD thesis, California Institute of Technology, http://resolver. caltech.edu/CaltechETD:etd-04092009-163047

Kumar, D., \& Bellan, P. M. 2006, RScI, 77, 083503

Kumar, D., \& Bellan, P. M. 2009, PhRvL, 103, 105003

Lebedev, S. V., Ciardi, A., Ampleford, D. J., et al. 2005, MNRAS, 361, 97

Li, H., Lapenta, G., Finn, J. M., Li, S., \& Colgate, S. 2006, ApJ, 643, 92

Li, H., Lovelace, R. V. E., Finn, J. M., \& Colgate, S. A. 2001, ApJ, 561, 915

Li, S., \& Li, H. 2003, Los Alamos National Lab. Tech. Rep. LA-UR-03-8935

Lovelace, R. V. E., Li, H., Koldoba, A. V., Ustyugova, G. V., \& Romanova, M. M. 2002, ApJ, 572, 445

Lynden-Bell, D. 1996, MNRAS, 279, 389

Lynden-Bell, D. 2003, MNRAS, 341, 1360

Moser, A. L. 2012, PhD thesis, California Institute of Technology, http://resolver.caltech.edu/CaltechTHESIS:04132012-150652134

Moser, A. L., \& Bellan, P. M. 2012a, Natur, 482, 379

Moser, A. L., \& Bellan, P. M. 2012b, Ap\&SS, 337, 593

Nakamura, M., Li, H., \& Li, S. 2006, ApJ, 652, 1059

Nakamura, M., Li, H., \& Li, S. 2007, ApJ, 656, 721

Nakamura, M., Tregillis, I. L., Li, H., \& Li, S. 2008, ApJ, 686, 843

Ray, T. P., Muxlow, T. W. B., Axon, D. J., et al. 1997, Natur, 385, 415

Romero-Talamás, C. A., Bellan, P. M., \& Hsu, S. C. 2004, RScI, 75, 2664

Shang, H., Allen, A., Li, Z.-Y., et al. 2006, ApJ, 649, 845

Sherwin, B. D., \& Lynden-Bell, D. 2007, MNRAS, 378, 409

Shikama, T., \& Bellan, P. M. 2013, RScI, 84, 023507

Shu, F. H., Najita, J., Ostriker, E. C., \& Shang, H. 1995, ApJL, 455, L155

Spruit, H. C. 2010, in The Jet Paradigm Lecture Notes in Physics, Vol. 794, ed. T. Belloni (Berlin: Springer), 233

Stenson, E. V., \& Bellan, P. M. 2012, PhRvL, 109, 075001

Uzdensky, D. A., \& MacFadyen, A. I. 2006, ApJ, 669, 546

Xu, H., Li, H., Collins, D., Li, S., \& Norman, M. L. 2008, ApJL, 681, L61

Yun, G. S., \& Bellan, P. M. 2010, PhPl, 17, 062108

Yun, G. S., You, S., \& Bellan, P. M. 2007, NucFu, 47, 181

Zavala, R. T., \& Taylor, G. B. 2005, ApJL, 626, L73 